\title{
Prior audio-visual learning facilitates auditory-only speech and voice-identity recognition in noisy listening conditions
}

\author{
Corrina Maguinness ${ }^{* 1,2}$, Sonja Schall2 ${ }^{2}$ \& Katharina von Kriegstein ${ }^{1,2}$ \\ 1 Chair of Cognitive and Clinical Neuroscience, Technische Universität Dresden, Dresden, Germany \\ 2 Max Planck Institute for Human Cognitive and Brain Sciences, Leipzig, Germany \\ *corrina.maguinness@tu-dresden.de; sonjaschall@gmail.com; \\ katharina.von kriegstein@tu-dresden.de \\ *Corresponding author
}

\begin{abstract}
Perception of human communication signals is often more robust when there is concurrent input from the auditory and visual sensory modality. For instance, seeing the dynamic articulatory movements of a speaker, in addition to hearing their voice, can help with understanding what is said. This is particularly evident in noisy listening conditions. Even in the absence of concurrent visual input, visual mechanisms continue to be recruited to optimise auditory processing: auditory-only speech and voice-identity recognition is superior for speakers who have been previously learned with their corresponding face, in comparison to an audio-visual control condition; an effect termed the "face-benefit". Whether the face-benefit can assist in maintaining robust perception in noisy listening conditions, in a similar manner to concurrent visual input, is currently unknown. Here, in two behavioural experiments, we explicitly examined this hypothesis. In each experiment, participants learned a series of speakers' voices together with their corresponding dynamic face, or a visual control image depicting the speaker's occupation. Following learning, participants listened to auditory-only sentences spoken by the same speakers and were asked to recognise the content of the sentences (i.e., speech recognition, Experiment 1 ) or the identity of the speaker (i.e., voice-identity recognition, Experiment 2 ) in different levels of increasing auditory noise (SNR $+4 \mathrm{~dB}$ to $-8 \mathrm{~dB}$ ). For both speech and voiceidentity recognition, we observed that for participants who showed a face-benefit, the benefit increased with the degree of noise in the auditory signal (Experiment 1,2). Taken together, these results support an audio-visual model of human auditory communication and suggest that the brain has developed a flexible system to deal with auditory uncertainty - learned visual mechanisms are recruited to enhance the recognition of the auditory signal.
\end{abstract}

Keywords: voice-identity, speaker-identity, speech, noise, audio-visual, multisensory

\section{Introduction}

Although speech is primarily conveyed acoustically, there is a large body of evidence that speech recognition is improved when the listener can both hear and see the speaker (see Peelle and Sommers, 2015 for review). These improvements are particularly apparent when the auditory signal is degraded, for example, in the presence of acoustic noise (Sumby and Pollack, 1954; Erber, 1969; Rosenblum, Johnson and Saldana, 1996; 
Grant and Seitz, 2000; Ross et al., 2007). Studies which have parametrically altered the noise level in the auditory signal have observed that the benefit of concurrent perception of visual, in addition to auditory, speech input increases in low auditory signal-to-noise ratios (SNR) (Sumby and Pollack, 1954; Erber, 1969; Ross et al., 2007), with a maximum benefit observed at specific SNRs (e.g., -12 dB - Ross et al., 2007; Liu et al., 2013). This visual benefit is thought to occur because the speaker's dynamic articulatory movements are correlated with the production of the auditory speech signal (Rosenblum, Johnson and Saldana, 1996; Campbell, 2008; Chandrasekaran et al., 2009; Scholes, Skipper and Johnston, 2020). Thus, they can assist in generating predictions about what is to be heard in the auditory speech signal (Jääskeläinen et al., 2004; Van Wassenhove, Grant and Poeppel, 2005; Davis et al., 2008; Arnal et al., 2009; Maguinness et al., 2011; Chandrasekaran and Ghazanfar, 2013; Peelle and Sommers, 2015; Bourguignon et al., 2020), enhancing speech recognition in these challenging listening conditions (Figure 1A).

Perhaps surprisingly, and contrary to conventional views of auditory processing (Ellis, Jones and Mosdell, 1997; Hickok and Poeppel, 2007; review see von Kriegstein, 2012) (Figure 1B), there is evidence that the sensory system continues to exploit visual mechanisms under auditory-only listening conditions (Figure 1C). Listeners are more accurate at recognising the auditory-only speech utterances of speakers who have been previously learned with their corresponding face, in comparison to an audio-visual control condition (von Kriegstein et al., 2008; Schelinski, Riedel and von Kriegstein, 2014; Riedel et al., 2015). In parallel, another aspect of auditory communication - voiceidentity recognition (also referred to as speaker recognition) - is enhanced following audio-visual voice-face learning. Listeners are superior at recognising the identity of voices that have been previously learned together with the speaker's face, in comparison to several auditory-only and audio-visual control learning conditions (Sheffert and Olson, 2004; von Kriegstein and Giraud, 2006; von Kriegstein et al., 2008; Schall et al., 2013; Schelinski, Riedel and von Kriegstein, 2014; Zäske, Mühl and Schweinberger, 2015). The behavioural enhancement seen for both speech and voice-identity recognition in auditory-only conditions has been termed the "face-benefit" (von Kriegstein et al., 2008). In both instances, it emerges rapidly following circa two-minutes audio-visual exposure with the speaker's identity (von Kriegstein et al., 2008; Schall et al., 2013; Schelinski, Riedel and von Kriegstein, 2014) and is observed in the majority of neurotypical participants (i.e., $76 \%$ for voice-identity and 58\% for speech recognition; von Kriegstein et al., 2008).

The face-benefits for speech and voice-identity recognition have been explained under an audio-visual model of human auditory communication (von Kriegstein et al., 2005, 2008; von Kriegstein and Giraud, 2006; review see von Kriegstein, 2012) (Figure 1C). In this view, face-benefits are driven by a generative model (Kiebel, Daunizeau and Friston, 2009) of the learned speaker. Specifically, a face-benefit on auditory-only processing likely arises because, during audio-visual exposure, sensory processing becomes rapidly tuned to the spatio-temporal relationship between the auditory speech 
signal and the dynamic (e.g., lip movements) and static (i.e., structural form) face features, in a speaker-specific manner (von Kriegstein et al., 2008). Subsequently, even in the absence of concurrent visual input, the sensory system can exploit this learned non-arbitrary sensory coupling: It simulates the missing dynamic visual trajectory of the auditory signal for speech recognition and the static face features for voice-identity recognition.

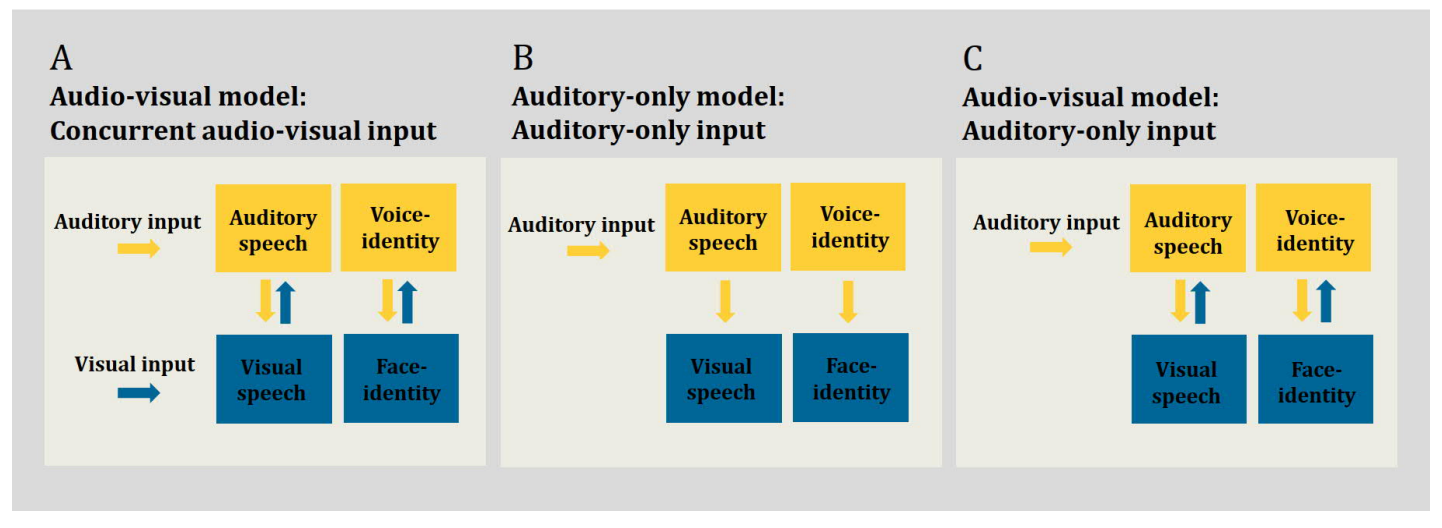

Figure 1. Schematic overview of models of human auditory communication. A) Audio-Visual Model: Concurrent Audio-Visual Input. Processing of the auditory signal (auditory speech and voice-identity) is supported by interactions (indicated via bidirectional arrows) between the visual and auditory systems (Peelle and Sommers, 2015; Young, Frühholz and Schweinberger, 2020). Here, concurrent visual cues help to predict and enhance the sensory processing of the auditory signal, this is particularly beneficial in noisy listening conditions (Sumby and Pollack, 1954). B) Auditory-Only Model: Auditory-Only Input. The auditory system is engaged in the sensory processing of the auditory signal (auditory speech and voiceidentity)(Ellis, Jones and Mosdell, 1997; Hickok and Poeppel, 2007). Any engagement of the visual system, including for speakers known by face, is epiphenomenal to the successfully processed auditory signal (indicated via unidirectional arrows) (Bunzeck et al., 2005). Under this model, learned visual mechanisms are not behaviourally relevant for auditory-only processing and, consequently, would not benefit sensory processing in noisy listening conditions. C) Audio-Visual Model: Auditory-Only Input. Both the auditory and visual system are engaged in the sensory processing of the auditory signal (auditory speech and voiceidentity) (von Kriegstein et al., 2008; von Kriegstein, 2012). Interactions between the systems (indicated via bidirectional arrows) are behaviourally relevant: in a similar manner to concurrent visual input (see panel A), learned visual mechanisms (for speakers known by face) assist in auditory processing by generating predictions and providing constraints about what is to be heard in the auditory signal (von Kriegstein et al., 2008), such a process should be particularly beneficial in noisy listening conditions. In all three models, yellow colours denote the auditory system, blue colours the visual.

In accordance with this model (Figure 1C), neuroimaging studies demonstrate that the left posterior superior temporal sulcus motion-sensitive face area (pSTS-mFA) is recruited during the recognition of auditory-only speech utterances of face learned speakers (von Kriegstein et al., 2008). Inhibitory stimulation of the pSTS-mFA reduces the face-benefit for auditory-only speech recognition (Riedel et al., 2015). Conversely, responses in the fusiform face area (FFA) - a region implicated in processing static structural face cues which support face-identity processing (Kanwisher, McDermott and Chun, 1997; Eger, Schyns and Kleinschmidt, 2004; Grill-Spector, Knouf and Kanwisher, 2004; Rotshtein et al., 2005; Kanwisher and Yovel, 2006; Ewbank and Andrews, 2008; Liu, Harris and Kanwisher, 2010; Axelrod and Yovel, 2015; Weibert and Andrews, 2015) - are apparent during the recognition of speakers known by face (von Kriegstein and Giraud, 2006; von Kriegstein et al., 2008; Schall et al., 2013). These FFA responses are 
behaviourally relevant: they positively correlate with the amount of face-benefit for voice-identity recognition (von Kriegstein et al., 2008). Cross-modal interactions between the visual face processing regions (left pSTS-mFA, FFA) and the respective auditory regions (left anterior superior temporal gyrus/ sulcus (aSTG/ S), temporal voice areas (TVA) in the right STG/ S) are also readily observed during auditory-only speech and voice-identity processing (von Kriegstein and Giraud, 2006; von Kriegstein, Kleinschmidt and Giraud, 2006; Schall and von Kriegstein, 2014).

While the visual mechanisms which underpin the face-benefit for speech and voice-identity recognition are thought to be distinct (i.e., dynamic cues for speech and static for identity), the governing principles for such cross-modal interactions are assumed to be the same: Visual mechanisms assist subsequent auditory-only recognition by generating predictions about, and thus placing constraints on the processing of, the incoming auditory signal (von Kriegstein et al., 2008; Blank, Kiebel and von Kriegstein, 2015). Such a process would be particularly beneficial for optimising recognition when the auditory signal is weak or degraded, with predictions assisting recognition by "filling in" missing sensory information. Currently, however, it is unknown whether in a similar vein to concurrent visual input ( Sumby and Pollack, 1954; Ross et al., 2007) the face-benefits for speech and voice-identity recognition are evident in noisy listening conditions and scale with increasing noise in the auditory signal. The aim of the studies presented here was to test this hypothesis. Such an observation would provide critical support for an audio-visual model of human auditory communication by demonstrating that visual mechanisms play a similar role to concurrent visual input in supporting auditory-only processing (Figure 1C).

To test our hypothesis, we conducted two behavioural experiments in which we examined the impact of audio-visual exposure on subsequent auditory-only recognition in noisy listening conditions. We adopted a parametric design similar to previous reports on the effect of concurrent auditory and visual speech inputs (e.g., Sumby and Pollack, 1954; Erber, 1969; Ross et al., 2007; Liu et al., 2013). We tested whether the face-benefit increased with decreasing SNRs of the auditory signal. In Experiment 1 and 2, we examined the face-benefit for auditory-only speech (Experiment 1) and voiceidentity (Experiment 2) recognition performance in four levels of auditory noise (SNRs: $+4 \mathrm{~dB}, 0 \mathrm{~dB},-4 \mathrm{~dB},-8 \mathrm{~dB}$ ). In both experiments the speakers were learned prior to auditory-only testing. Crucially, half of the speakers were learned by seeing and listening to videos of the speaker talking (voice-face learning). The other half were learned by listening to the speaker while viewing a visual control image depicting the speaker's occupation (voice-occupation learning). Our first and central hypothesis was that the face-benefit would increase with decreasing SNRs in the auditory signal. Such a finding would indicate that learned visual mechanisms can help to systematically resolve incoming noisy auditory input. We expected this effect to be evident for both speech (Experiment 1) and voice-identity (Experiment 2) recognition. In addition, replicating previous findings (Sheffert and Olson, 2004; von Kriegstein et al., 2008; Schall et al., 2013; Schelinski, Riedel and von Kriegstein, 2014), we expected to observe a general 
benefit for audio-visual learning on speech and voice-identity recognition i.e., the facebenefit would be present regardless of noise level tested.

\section{Experiment 1}

\section{Materials and Methods}

\section{Participants}

Thirty-seven, right handed (Oldfield, 1971), native German speakers, recruited from the participant database of the Max Planck Institute for Cognitive and Brain Sciences (Leipzig, Germany) and the participant management system of the Technische Universität Dresden (Dresden, Germany) took part in this experiment. All participants reported normal, or corrected to normal, vision and normal hearing. All participants gave their informed written consent prior to participation. Seven of the participants failed to reach the threshold of $\geq 80 \%$ correct during the audio-visual training stage (see section Audio-visual training and auditory-only speech recognition below) and therefore did not proceed to the auditory-only test phase of the experiment. Analyses was based on thirty participants ( 13 female, mean age $=24.8$ years; $\mathrm{SD}=2.6$ years).

\section{Stimuli and Apparatus}

Audio-visual training and auditory-only speech recognition

The stimuli consisted of auditory and video recordings of six male speakers (2230 years old). The auditory stimuli were recorded in a soundproof room using high quality recording equipment (Microphone: TLM 50, Neumann, Berlin; Mic-Preamp: MicAmp F35, Lake People, Germany; Soundcard: Power Mac G5 Dual 1.8 GHz, Apple Inc., CA, USA; Software: Sound Studio 3, Felt Tip, Inc., NY, USA) (44.1 kHz; 16 bit, mono). Videos were recorded using a high-definition camera (HDLegria HF S10, Canon, Japan) and an external microphone. The auditory stimuli were adjusted for overall mean amplitude using Matlab7 (MathWorks, MA, USA). Video stimuli were edited in Final Cut Pro software (Apple Inc., California, USA) to include a circular centered mask, which excluded the background while revealing the face of the speaker. Videos were cropped to $727 \times 545$ pixels. In addition, for each speaker a single frame was extracted from the video sequence. The image contained the speaker in a neutral non-speech pose, i.e., with the mouth closed. Three symbols representing an occupation were taken from Clip Art (http://office.microsoft.com/en-us/). The stimuli for the audio-visual voice-face training phase consisted of five video and three auditory recordings of questions which were five- to six-words in length and each approximately 2.5 seconds in duration (e.g., “Trägt der Junge einen Koffer?”, English: “Is the boy carrying a suitcase?”). The stimuli for the audio-visual voice-occupation training phase were identical to that used for the voice-face training with the exception that the dynamic video image was replaced with 
one of the Clip Art pictures. Sentences used in the auditory-only speech recognition test phase were 30 statement sentences which were five- to six-words in length, (e.g., "Der Mann geht am Stock", English: "The man walks with a cane"), each with a mean duration of approximately 2.5 seconds. These auditory-only test stimuli (presented at $65 \mathrm{~dB}$ SPL) were mixed with pink noise (created in Matlab7 by filtering Gaussian white noise) of varying intensities to produce signal-to-noise ratios of $-8,-4,0$, and $+4 \mathrm{~dB}$. These SNRs and intervals (i.e., - $4 \mathrm{~dB}$ steps) are comparable to previous audio-visual speech-in-noise studies (0dB, $-4 \mathrm{~dB},-8 \mathrm{~dB}$ - see Ross et al., 2007; Liu et al., 2013). Based on pilot testing in a different participant sample $(\mathrm{N}=6)$, we did not include lower $\mathrm{SNRs}$ as they produced close to chance performance ( $\mathrm{SNR}-10 \mathrm{~dB}, \mathrm{M}=57 \%$ ). The pilot testing used a similar task design as the main experiment, but participants were exposed to only one noise level during auditory-only testing. The SNR of $+4 \mathrm{~dB}$ was included to replicate previous studies on the face-benefit which used a SNR of $+3 \mathrm{~dB}$ (Schall et al., 2013; Schelinski, Riedel and von Kriegstein, 2014; Riedel et al., 2015). The pink noise was ramped and introduced with a linear 50ms fade-in and fade-out. The experiment was programmed in and run using Presentation software (Neurobehavioral Systems, Inc., California, USA). It was presented on a 19" Samsung Syncmaster CRT monitor (Samsung Electronics Co., Ltd., Suwon, South Korea). All auditory stimuli were presented binaurally via Sennheiser HD280pro headphones (Sennheiser Electronic GmbH \& Co. KG, Hannover, Germany).

\section{Design}

The experiment was based on a within-subject factorial design with Learning (voice-face, voice-occupation) and Noise Level $(-8,-4,0$, and $+4 \mathrm{~dB})$ as repeated factors. Learning was implemented using an established audio-visual training protocol which has demonstrated the face-benefit in previous studies (von Kriegstein et al., 2008; Schall et al., 2013). As we were specifically interested in the effect of noise level on the facebenefit, we kept the learning design as comparable as possible to the previous studies. The dependent variable was response accuracy for recognising auditory-only speech utterances, for voice-face, relative to voice-occupation, learned speakers. To investigate a potential speed-accuracy trade-off we also analysed response times.

Procedure

Audio-visual training

All participants were familiarised with the same six speakers. Participants learned three of the speakers together with their corresponding face-identity (voiceface learning) and three with a visual symbol depicting their occupation (voiceoccupation learning). In both conditions the participant also learned the speaker's name (Figure 2A). The three speakers assigned to each learning condition were counterbalanced across participants. We used these two learning conditions because they have been shown to reveal a replicable face-benefit across studies (von Kriegstein et al., 2008; Schelinski, Riedel and von Kriegstein, 2014). 
Familiarisation with the speakers was achieved through a number of training rounds. Each round consisted of a learning stage, followed by an evaluation stage. In the learning stage, participants were exposed to two blocks of audio-visual trials containing the five- to six-word question clips of speakers paired with either their corresponding face-identity (voice-face learning) or with an occupation symbol (voice-occupation learning). The speaker sets, i.e., voice-face or voice-occupation, were always presented in separate blocks and the starting order of voice-face and voice-occupation learning was counterbalanced across participants. Each block consisted of 3 speakers uttering 5 five- to six-word sentences. The name of the speaker appeared onscreen before the audio-visual speaker was presented. Participants were instructed to make a good impression of the names, voices and faces/occupations, but not to respond. Each speaker was heard saying each sentence once, amounting to 15 trials per block. Trials within a block were presented in a randomised order and were interleaved with an inter-trial-interval of 1 second.

Learning was assessed after each audio-visual speaker block using a response active evaluation stage. Here, participants completed 18 trials in which they had to match the name to the voice or the face/ occupation of the 3 speakers from the preceding block. In each trial participants listened to one of three sentences (five- to six-word questions), which was immediately followed by either a name or a face/ occupation image. Each speaker was heard uttering each of the three sentences two times, amounting to 6 trials per speaker. Sentences used in the evaluation stage were not presented during audio-visual learning. On half of the trials the voice matched the name or image, while the remaining trials contained mismatched, i.e., distractor, names/images. The distractor names and images were always taken from the same speaker set (i.e., voice-face or voice-occupation). Participants indicated via button-press whether the name and the voice or face/ occupation belonged to the same speaker ("yes" or "no"). The name or image remained onscreen until a response was made. Participants received written onscreen feedback (correct/ incorrect) for $500 \mathrm{~ms}$ after a response was made. This was followed by a presentation of the correct name and voice-face or voiceoccupation pairing. All participants completed at least two rounds of training. If after two rounds participants could reliably match the correct combinations of voice, name, and face/ occupation (criterion: $\geq 80 \%$ correct), they began the auditory-only speech recognition test stage (see next section). Thus, training consisted of 60 learning trials (15 trials per speaker $\mathrm{x} 4$ blocks) and evaluation of 72 trials (18 trials per speaker $\mathrm{x} 4$ blocks) for participants who needed two rounds to reach the criterion $(\mathrm{N}=30)$. Those with $<80 \%$ correct completed another training round, if they failed to reach the criterion upon completing the third training round, they were excluded from the experiment and did not complete the recognition test phase. This was the case for seven participants.

Auditory-only speech recognition

In the auditory-only speech recognition test, participants listened to sentences uttered by the same six speakers who had been learned during the audio-visual training 
phase. In each trial, participants viewed a white fixation cross, presented at the center of the screen, while they listened to a speaker utter a five- to six-word statement sentence. The sentence was followed by the presentation of a visual word onscreen. The participant was instructed to indicate via button press, "yes" or "no", whether the word

\section{A. Audio-visual training}
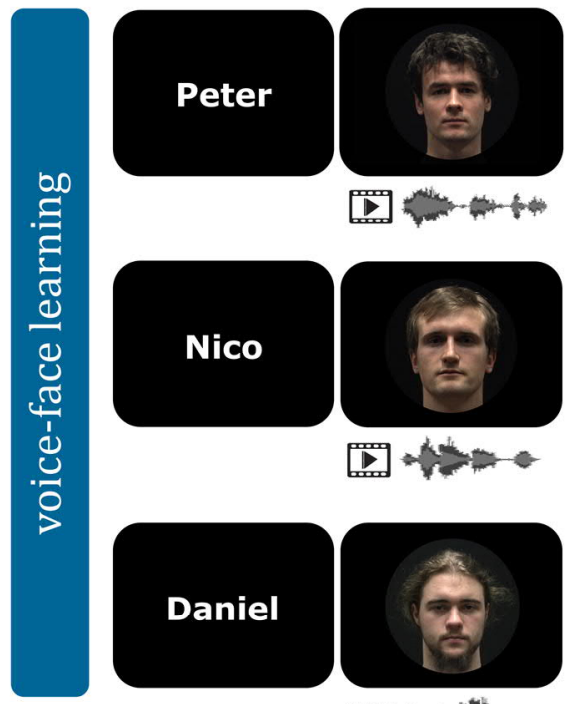

睓
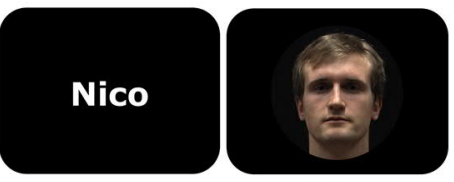

䍘
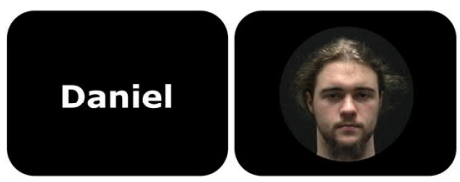

$1 \mathrm{sec}$
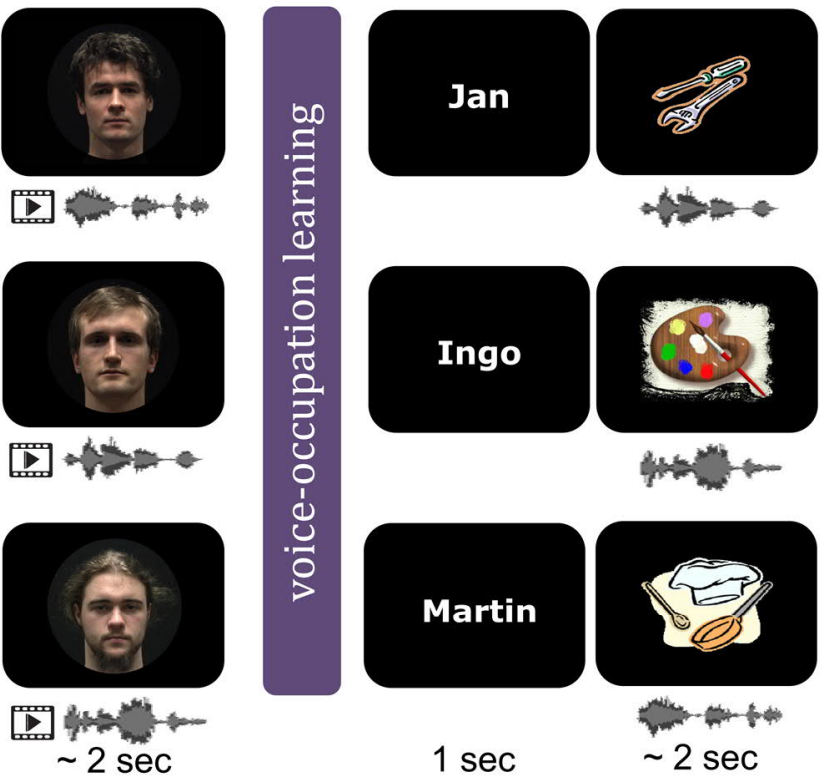

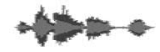
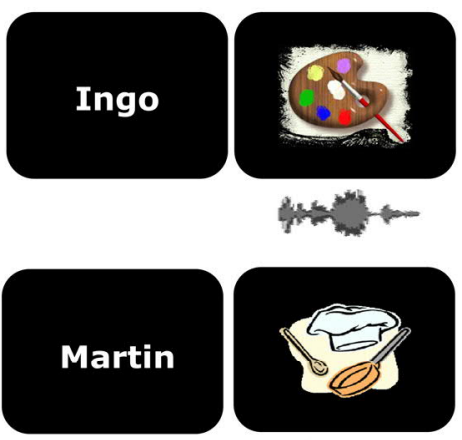

$1 \mathrm{sec}$

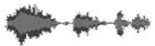

$\sim 2 \mathrm{sec}$

\section{B. Auditory-only speech recognition (sample trials)}

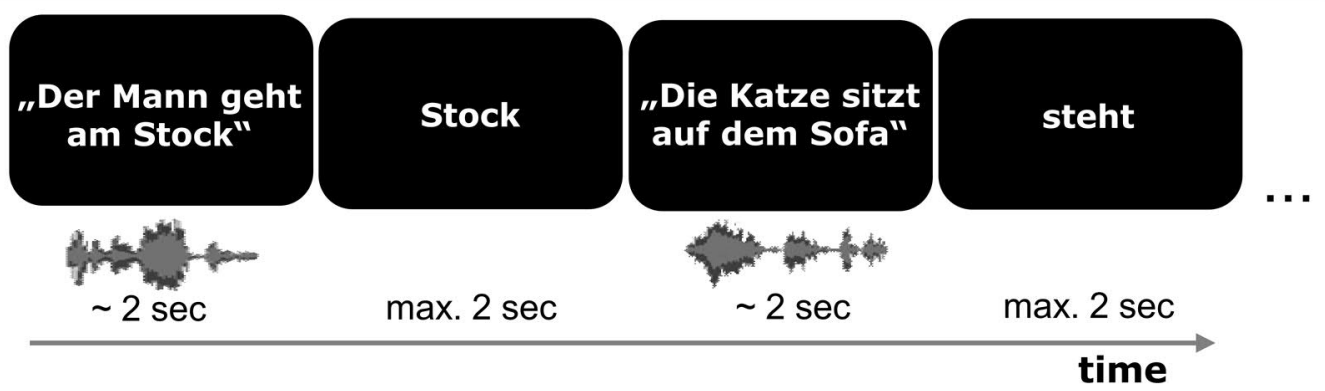

Figure 2. Schematic Illustration of the audio-visual training phase and auditory-only speech recognition test phase in Experiment 1. (A) Audio-visual training. Prior to auditory-only testing, participants learned the voice and name of six speakers in conjunction with their corresponding face, i.e., video (voice-face learning) or with an occupation image (voice-occupation learning). (B) Auditory-only speech recognition. Following audio-visual training, participants listened to auditory-only sentences spoken by the same learned six speakers in different levels of auditory noise $(-8,-4,0,+4 \mathrm{~dB})$. The sentences were presented in blocks (15 trials per block), which were blocked by learning type (voice-face or voiceoccupation) and noise level. In each trial, participants listened to a speaker utter a five- or six-word sentence. This was followed immediately by the presentation of a word onscreen. The participant's task was to decide if the presented word was contained within the previously heard sentence or not. 
was present in the preceding sentence. The word was displayed until a response was made, up to a maximum of 2 seconds (Figure 2B). A response triggered the next trial (or the next trial began after 2 seconds if there was no response). In half of the trials the displayed word was a correct match for a word which had occurred within the sentence. In the remaining trials the word was an incorrect match. Words for the incorrect trials were chosen for either their semantic or phonological similarity to those in the sentences. For example, for the sentence "Der Mann geht am Stock", English: "The man walks with a cane" the presented correct words could be "Mann", "geht" or "Stock" and for the incorrect words "Dock", "steht" or "Kranke". The sentences were presented in four different levels of auditory noise $(-8 \mathrm{~dB},-4 \mathrm{~dB}, 0 \mathrm{~dB}$ and $+4 \mathrm{~dB})$. There were 720 trials in total: each of the 6 speakers uttered each of the 30 sentences once in four levels of auditory noise ( $6 \times 30 \times 4)$. Trials were presented in 48 separate blocks, containing 15 trials. Each block was grouped by speaker type (i.e., voice-face or voice-occupation) and speaker identities within a block were presented in a randomised order. Sentences in each block were drawn randomly from the 30-sentence set. Each block contained one level of auditory noise $(-8 \mathrm{~dB},-4 \mathrm{~dB}, 0 \mathrm{~dB}$ or $+4 \mathrm{~dB})$. The block order of noise-levels was randomised throughout. After 12 blocks the participants were given a rest period of 60 seconds.

A brief practice period proceeded the auditory-only speech recognition test. To refresh participants' memory of the speakers, participants were presented with one audio-visual presentation of each speaker and their corresponding name, in the absence of auditory noise. This was followed by six practice trials to familiarise the participant with the task and introduce them to the auditory noise. In the practice trials, the participant recognised the speech utterances of the six learned speakers in the highest level of auditory noise (procedure as in the main task). Participants could repeat these six practice trials until they were comfortable with the testing procedure.

\section{Data Analysis}

Trials in which the participant failed to make a response, i.e., missed trials, were disregarded from analysis (total $0.3 \%$ of all trials across participants, std. dev. 0.4\%; min. $\%$ of missed trials was $0 \%$, the max. was $1.67 \%$ ). The overall trial count was then adjusted to include only trials for which a response was made. Accuracy was calculated as the number of correctly identified words divided by the adjusted trial count, for each participant, for each condition i.e., voice-face and voice-occupation learning conditions, per noise level. Reaction times (in milliseconds) were also calculated for correct response trials, for each participant, for each condition. Mean accuracy and reaction time data can be viewed in Table 1.

To test our central hypothesis of whether the face-benefit increased with noise level, we calculated a face-benefit score per participant using the accuracy data: (\% correct voice-face learning, minus, \% correct voice-occupation learning), per noise level. This score reflects how much a participant benefits, in terms of speech recognition 
accuracy performance, from learning a voice with its corresponding face counterpart (von Kriegstein et al., 2008). Based on observations that the benefit of concurrent visual input on speech processing can scale with increasing noise and peaks at -12 dB SNR (Ross et al., 2007), we tested for a linear relationship with the face-benefit score across noise levels using a simple linear regression, with the noise level as a predictor variable and the face-benefit as the dependent variable. We also tested if the face-benefit was evident in each of the separate noise levels using one-sample t tests (comparing the facebenefit scores to zero i.e., no face-benefit) corrected for multiple comparisons. In addition to the face-benefit analyses on accuracy, we also examined reaction time (RT) data using the same procedure i.e., calculating a face-benefit RT per participant, per noise level (negative scores indicating faster responses for voice-face learned speakers), and analysed the RT using a simple linear regression and one-sample t tests. This supplementary analysis served to determine if speech recognition was superior, in terms of both accuracy and reaction time (i.e., no speed accuracy trade off), for voices learned together with their face, compared to the audio-visual control condition. For all analyses (Experiment 1, 2), the $\alpha$ level for significance was defined at 5\% (HolmBonferroni adjusted for multiple comparisons (Holm, 1979), where appropriate). Effect sizes were calculated using partial eta square $\eta_{p}^{2}$ (Cohen, 1969; Richardson, 2011) and Cohen's d (Cohen, 1988) for t tests. All t tests were calculated two-tailed. All data analyses were carried out using Statistica (TIBCO Software, www.tibco.com).

\section{Results}

\section{Face-Benefit Score: Accuracy}

\section{Face-Benefit: Across Noise Levels}

To test our first hypothesis, we examined evidence for a linear relationship between the face-benefit for speech recognition and increasing auditory noise. At a group level $(\mathrm{N}=30)$, there was no evidence of a correlation between the face-benefit and noise level $(r=0.08, p=0.40)$. The linear regression results demonstrated that noise level did not significantly predict face-benefit scores $(\beta=0.08, t(118)=0.84, p=0.40)$ or explain a significant proportion of the variance in the face-benefit scores $\left(\mathrm{R}^{2}=0.006\right.$, $\left.F(1,118)=0.71, p=0.40, n_{p}^{2}=0.006\right)$. Next, as our main objective was to observe the change in the face-benefit as the noise-level increased, we restricted our analysis to include only the participants who benefitted from voice-face learning. In line with previous observations, we observed an overall benefit (i.e., average positive score across noise levels) of voice-face learning in a subsample, 14 of 30 (47\%), of the participants tested. This percentage is similar to previous observations for speech recognition (von Kriegstein et al., 2008). In line with our predictions, in these participants, there was a significant negative correlation between the face-benefit and noise level $(r=-0.42, p=$ $0.001)$. The face-benefit increased with decreasing signal-to-noise ratios. The results of the linear regression demonstrated that noise level significantly predicted face-benefit scores $(ß=-0.42, t(54)=-3.40, p=0.001)$. The model results also showed that noise 
level explained $18 \%$ of the variance in the face-benefit scores $\left(R^{2}=0.18, F(1,54)=11.55\right.$, $\left.p=0.001, \eta_{p}{ }^{2}=0.18\right)$. On examination of Figure $3 \mathrm{~A}$, one can see that this effect appears to be driven by a large increase in the face-benefit at SNR $-8 \mathrm{~dB}$ i.e., in the noisiest listening condition.

We noted that the degree of variability in the face-benefit across individuals was large. For example, for SNR -8 dB face-benefit scores ranged from 0 to 14\% (Figure 3A). We therefore also calculated a normalised face-benefit score: this was defined as the difference in percent correct across voice-face and voice-occupation learned speakers for each noise level in each individual, divided by the individual's overall face-benefit score i.e., the average across all noise levels. This score more accurately reflects the relative face-benefit in speech recognition as it considers the face-benefit score, in a given noise level, as a consequence of the overall face-benefit score. We repeated the linear analysis using this normalised score, again restricting the analysis to only those with a positive overall face-benefit. Note that inclusion of participants with a negative face-benefit score would produce a false positive normalised face-benefit score i.e., dividing the negative scores by a negative value. Again, in line with our central hypothesis, we confirmed the results of the previous analysis. The normalised facebenefit and noise level were significantly negatively correlated $(r=-0.39, p=0.003$; Figure 3B). The results of the model for the linear regression showed that the level of noise in the auditory signal significantly predicted $(\beta=-0.39, \mathrm{t}(54)=-3.15, \mathrm{p}=0.003)$ and explained a significant amount of the variance $(16 \%)$ in the normalised face-benefit scores $\left(R^{2}=0.16, F(1,54)=9.91, p=0.003, \eta_{p}^{2}=0.16\right)$. As per the previous analysis, when examining Figure 3B, this effect appeared to be largely driven by the increase in the normalised face-benefit at the lowest SNR tested (SNR -8 dB), while the amount of normalised face-benefit was relatively stable in the less noisy listening conditions.

\section{Auditory-Only Speech Recognition Accuracy}

A

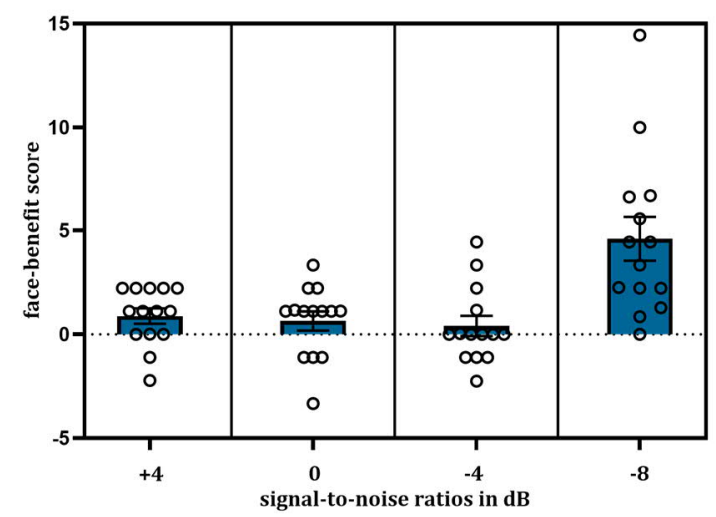

B

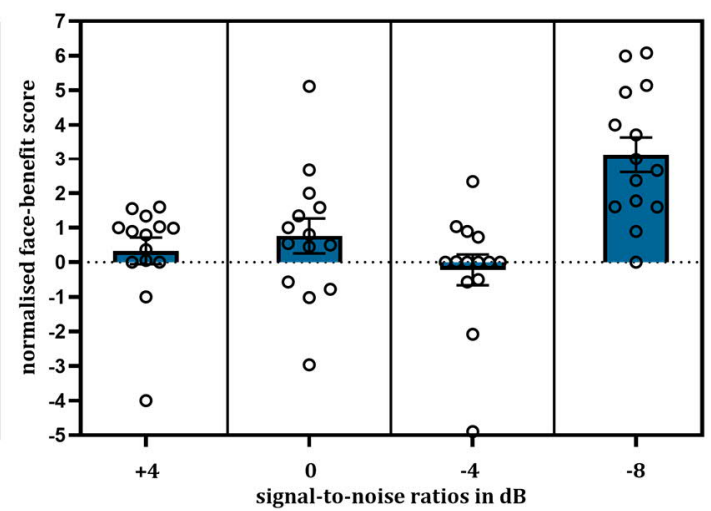

Figure 3. Plots showing the (A) face-benefit score and (B) normalised face-benefit score for speech recognition $(\mathrm{N}=14)$ with decreasing $\mathrm{SNRs}$ of the auditory signal. The (normalised) face-benefit was most evident in the noisiest listening condition - panel (A) and (B) SNR -8 dB ( $p \leq 0.007)$. In (A) and (B) error bars show \pm 1 standard error of the mean. Individual participant scores are displayed as single points. 
Face-Benefit: Per Noise Level

We also examined the face-benefit for speech recognition separately in each of the noise levels. For this categorical analysis, we used the face-benefit score per discrete noise level (i.e., the non-normalised score). At a group level $(\mathrm{N}=30)$, the face-benefit for speech recognition was not evident in any of the four noise levels (all ps >0.027; nonsignificant with Holm-Bonferroni correction for the 4 tests; Table 1). For the analysis including the 14 participants with a positive overall face-benefit, the face-benefit significantly differed from zero in the noisiest listening condition i.e., SNR -8 dB (M facebenefit $=4.6 \%)(t(13)=4.38, p=0.007,95 \%$ CI $[2.33,6.86], d=1.17)$. See Figure 3A. In all other listening conditions, when Holm-Bonferroni correcting for four comparisons, the face-benefit score did not significantly differ from zero - SNR $-4 \mathrm{~dB}(\mathrm{t}(13)=0.82, \mathrm{p}$ $=0.43,95 \%$ CI $[-0.66,1.46], \mathrm{d}=0.22)$; SNR $0 \mathrm{~dB}(\mathrm{t}(13)=1.38, \mathrm{p}=0.19,95 \%$ CI $[-0.36$, 1.64], $d=0.37) ; \mathrm{SNR}+4 \mathrm{~dB}(\mathrm{t}(13)=2.35, \mathrm{p}=0.035,95 \% \mathrm{CI}[0.07,1.68], \mathrm{d}=0.63)$. Figure $3 \mathrm{~A}$. Note this pattern of results remained for the analyses with the normalised facebenefit scores for $N=14$ : SNR $-8 d B(p<0.001)$, all other noise levels ( $p s>0.16)$. Figure 3B.

Table 1. Auditory-only speech recognition performance. Accuracy and reaction times scores (with standard deviations) for voice-face and voice-occupation learned speakers, in the four noise levels. Scores for all 30 participants are displayed on the left of the table, scores for the 14 participants with a positive overall face-benefit score (defined via accuracy) are to the right. All scores are derived from the nonnormalised data.

\begin{tabular}{|c|c|c|c|c|c|c|c|c|}
\hline \multirow[b]{2}{*}{ signal-to-noise ratio } & \multicolumn{4}{|c|}{$\begin{array}{l}\text { All Participants } \\
(\mathrm{N}=30)\end{array}$} & \multicolumn{4}{|c|}{$\begin{array}{l}\text { Positive Face-Benefit Participants } \\
(\mathrm{N}=14)\end{array}$} \\
\hline & $+4 \mathrm{~dB}$ & $\mathbf{0 ~ d B}$ & $-4 d B$ & $-8 \mathrm{~dB}$ & $+4 \mathrm{~dB}$ & $\mathbf{0 ~ d B}$ & $-4 d B$ & $-8 \mathrm{~dB}$ \\
\hline \multicolumn{9}{|l|}{ voice-face } \\
\hline$\%$ Correct & $\begin{array}{l}98.59 \\
(1.54)\end{array}$ & $\begin{array}{l}98.33 \\
(1.72)\end{array}$ & $\begin{array}{l}97.89 \\
(1.32)\end{array}$ & $\begin{array}{l}94.82 \\
(4.22)\end{array}$ & $\begin{array}{l}98.33 \\
(1.78)\end{array}$ & $\begin{array}{l}98.97 \\
(1.34)\end{array}$ & $\begin{array}{l}98.41 \\
(1.05)\end{array}$ & $\begin{array}{l}97.27 \\
(2.38)\end{array}$ \\
\hline RT (milliseconds) & $\begin{array}{l}669.89 \\
(99.69) \\
\end{array}$ & $\begin{array}{l}664.51 \\
(90.89)\end{array}$ & $\begin{array}{l}672.98 \\
(89.94)\end{array}$ & $\begin{array}{l}706.63 \\
(105.00)\end{array}$ & $\begin{array}{l}676.34 \\
(128.54)\end{array}$ & $\begin{array}{l}673.18 \\
(115.21)\end{array}$ & $\begin{array}{l}670.66 \\
(108.61)\end{array}$ & $\begin{array}{l}700.73 \\
(129.48)\end{array}$ \\
\hline \multicolumn{9}{|l|}{ voice-occupation } \\
\hline$\%$ Co & $\begin{array}{l}97.99 \\
(1.72)\end{array}$ & $\begin{array}{l}98.14 \\
(1.38)\end{array}$ & $\begin{array}{l}97.99 \\
(1.84)\end{array}$ & $\begin{array}{l}94.83 \\
(4.21)\end{array}$ & $\begin{array}{l}97.46 \\
(1.65)\end{array}$ & $\begin{array}{l}98.33 \\
(0.95)\end{array}$ & $\begin{array}{l}98.01 \\
(1.81)\end{array}$ & $\begin{array}{l}92.68 \\
(4.79)\end{array}$ \\
\hline RT (milliseconds) & $\begin{array}{l}671.65 \\
(91.59)\end{array}$ & $\begin{array}{l}675.32 \\
(93.57)\end{array}$ & $\begin{array}{l}675.79 \\
(96.27)\end{array}$ & $\begin{array}{l}697.07 \\
(87.91)\end{array}$ & $\begin{array}{l}669.55 \\
(111.67)\end{array}$ & $\begin{array}{l}684.60 \\
(114.71)\end{array}$ & $\begin{array}{l}693.48 \\
(118.05)\end{array}$ & $\begin{array}{l}704.78 \\
(112.12)\end{array}$ \\
\hline \multicolumn{9}{|l|}{ face-benefit } \\
\hline$\%$ Cor & $\begin{array}{l}0.60 \\
(1.40)\end{array}$ & $\begin{array}{l}0.19 \\
(1.68)\end{array}$ & $\begin{array}{l}-0.11 \\
(1.76)\end{array}$ & $\begin{array}{l}-0.01 \\
(5.51)\end{array}$ & $\begin{array}{l}0.87 \\
(1.39)\end{array}$ & $\begin{array}{l}0.64 \\
(1.73)\end{array}$ & $\begin{array}{l}0.40 \\
(1.83)\end{array}$ & $\begin{array}{l}4.59 \\
(3.92)\end{array}$ \\
\hline RT (milliseconds)* & $\begin{array}{l}-1.77 \\
(40.46)\end{array}$ & $\begin{array}{l}-10.82 \\
(41.07)\end{array}$ & $\begin{array}{l}-2.81 \\
(39.54)\end{array}$ & $\begin{array}{l}9.56 \\
(48.30)\end{array}$ & $\begin{array}{l}6.80 \\
(33.04)\end{array}$ & $\begin{array}{l}-11.42 \\
(30.85)\end{array}$ & $\begin{array}{l}-22.82 \\
(29.70)\end{array}$ & $\begin{array}{l}-4.06 \\
(31.90)\end{array}$ \\
\hline
\end{tabular}

*negative reaction times (RT) indicate comparatively faster responses.

Face-Benefit Score: Reaction Time

Face-Benefit Reaction Time: Across Noise Levels

The linear regression analysis including all 30 participants did not show any relationship between the face-benefit RT scores and the level of noise in the auditory 
signal $(\beta=-0.11, \mathrm{t}(118)=-1.21, \mathrm{p}=0.23)\left(\mathrm{R}^{2}=0.012, \mathrm{~F}(1,118)=1.46, \mathrm{p}=0.23, \mathrm{n}^{2}=\right.$ $0.012)$. Next, we examined reaction times for the participants who exhibited a positive overall face-benefit ( $\mathrm{N}=14$, based on accuracy performance). This analysis served to establish that the superior speech recognition across noise levels for these participants could not solely be explained by faster, and potentially less accurate, performance for voice-occupation learned speakers i.e., a speed-accuracy trade off. For these participants, there was also no relationship evident between face-benefit RTs and the noise level ( $r=0.15, p=0.26)$. The noise level did not significantly predict face-benefit RTs scores $(\beta=0.15, t(54)=1.14, p=0.26)$ or explain a significant amount of variance in these RTs $\left(R^{2}=0.023, F(1,54)=1.29, p=0.26, n_{p}{ }^{2}=0.023\right)$. This suggests no speed accuracy trade-off for the effect of learning, as a function of noise - i.e., reaction times were not systematically faster for voice-occupation, compared to voice-face, learned speakers. Rather RTs were qualitatively faster (reflected in negative RT values) for voice-face, compared to voice-occupation, learned speakers in several noise levels (Figure 4). The next analysis examined these categorical noise level RT effects.

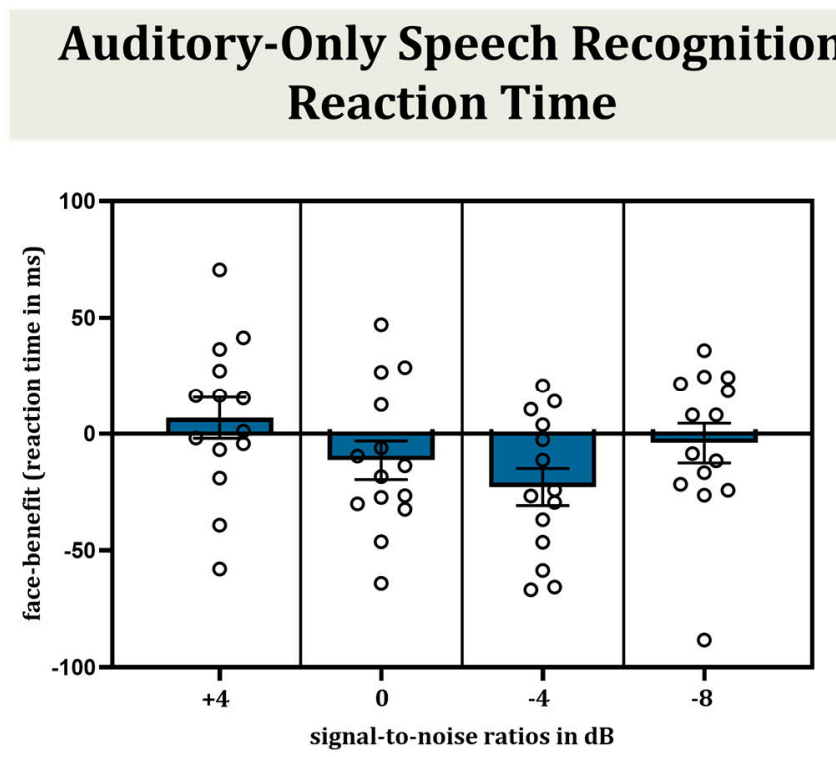

Figure 4. Plot of the face-benefit reaction time scores for speech recognition $(\mathrm{N}=14)$ for each of the SNR listening conditions. There was a trend for faster responses to voice-face, compared to voice-occupation, learned speakers in SNR -4 dB ( $p=0.013)$ - negative face-benefit reaction times indicate comparatively faster responses. Error bars show \pm 1 standard error of the mean. Individual participant scores are displayed as single points.

Face-Benefit Reaction Time: Per Noise Level

For the analyses examining the face-benefit RT scores in each separate noise level, for all $\mathrm{N}=30$, there was no significant face-benefit RT evident in any of the noise levels (all ps >0.16). For the analysis including the 14 participants with a positive overall face-benefit (based on accuracy), there was a trend, when Holm-Bonferroni correcting for four comparisons, that the face-benefit RT differed from zero in the SNR - $4 \mathrm{~dB}$ noise level ( $\mathrm{M}$ face-benefit RT $=-22.82$ milliseconds) $(\mathrm{t}(13)=-2.88, \mathrm{p}=0.013,95 \%$ CI [-39.97, 
-5.67], $d=-0.77$ )(Figure 4). In all other listening conditions the face-benefit score did not significantly differ from zero - SNR $-8 \mathrm{~dB}(\mathrm{t}(13)=-0.47, \mathrm{p}=0.62,95 \%$ CI $[-22.47$, 14.36], $\mathrm{d}=-0.13)$; SNR $0 \mathrm{~dB}(\mathrm{t}(13)=-1.39, \mathrm{p}=0.19,95 \%$ CI $[-29.23,6.39], \mathrm{d}=-0.37)$; $\mathrm{SNR}+4 \mathrm{~dB}(\mathrm{t}(13)=0.77, \mathrm{p}=0.46,95 \%$ CI $[-12.28,25.87], \mathrm{d}=0.21)$.

\section{Discussion}

The results revealed, in accordance with our hypothesis, that the (normalised) face-benefit increased with noise in the auditory signal. This was observed only for participants who exhibited an overall benefit for voice-face learning. Across the noise levels, this superior speech recognition performance could not be explained by reaction time differences when responding to voice-face learned speakers i.e., no speed/ accuracy trade off. While the linear analyses suggested that the (normalised) face-benefits scaled with decreasing SNRs, on visual examination of the plots one can see that this correlation was largely driven by the increase in the (normalised) face-benefit at the lowest SNR tested i.e., SNR -8. The categorical analysis, in each of the separate noise levels, also confirmed a significant (normalised) face-benefit in this noise level. In other less noisy listening conditions, a potential benefit of voice-face learning was rather reflected in reaction time performance, with qualitatively faster responses for speakers learned by face, than those learned by occupation.

The result that the face-benefit increases in noise, and that it is most apparent at a specific auditory SNR is similar to previous observations on speech processing in noise when concurrent visual input is available (Ross et al., 2007; Liu et al., 2013; Crosse, Di Liberto and Lalor, 2016). We will come back to this observation in the discussion of Experiment 2 and the main discussion. In Experiment 2, we turned to address whether the face-benefit in auditory-only voice-identity recognition also increases with decreasing SNRs.

\section{Experiment 2}

\section{Materials and Methods}

Participants

Thirty-four native German speakers, recruited from the Max Planck Institute for Cognitive and Brain Sciences (Leipzig) participant database, took part in this experiment. None of the participants had participated in Experiment 1. All were righthanded, as assessed by the Edinburgh Handedness Inventory (Oldfield, 1971) and reported normal hearing and normal, or corrected-to-normal, vision. All participants gave their informed written consent prior to participation. Seven of the participants failed to meet the learning criteria during the audio-visual training phase of the 
experiment ( $\geq 80 \%$ correct) and thus did not proceed to the auditory-only speaker recognition test phase. Additionally, one participant was excluded from analysis due to below chance performance in the auditory-only test phase of the experiment and another due to technical failure which disrupted data saving. As such, the analysis reported below is based on data from twenty-five participants ( 17 female, mean age $=$ 24.3 years; $\mathrm{SD}=2.3$ years).

\section{Stimuli and Apparatus}

Stimuli were audio and video recordings of six male German speakers. The stimuli were recorded and processed in the same manner as that reported in Experiment 1, with the exception that three of the speakers were from different male identities (age range of 6 speakers: 22-27 years old; set as in Schall et al., 2013, 2014). The stimuli for the audio-visual voice-face training comprised of five videos and three auditory recordings of five- to six-word statement sentences with a mean length of 2.5 seconds (e.g., "Die Rose wächst im Garten", English: "The rose grows in the garden"). Stimuli for the audio-visual voice-occupation training phase were identical to that of the voice-face training, with the exception that the visual video display was replaced with a static occupation symbol, shown for the same duration as the video sequence (i.e., similar to Experiment 1, see Figure 2A). Stimuli for the auditory-only voice-identity recognition test phase of the experiment consisted of 30 two-word sentences, always starting with "Er" (English: "He") and finishing with a verb (e.g. "Er liest", English: "He reads"). All sentences were approximately 1 second in length. Note these shorter sentences are sufficient for voice-identity tasks (Schall et al., 2013, 2014). As Experiment 1, auditory-only test stimuli were mixed with pink noise of varying intensities to produce signal-to-noise ratios (SNR) of $-8,-4,0$, and $+4 \mathrm{~dB}$. This noise mixing procedure and the apparatus used for stimulus presentation were the same as in Experiment 1.

\section{Design}

The design was the same as in Experiment 1, with the exception that the dependent variable was response accuracy and reaction times for auditory-only voiceidentity recognition for speakers learned by face, relative to those learned with an occupation symbol.

\section{Procedure}

\section{Audio-visual training}

The audio-visual training procedure, including learning and evaluation phases, was the same as in Experiment 1. 
Auditory-only voice-identity recognition

In the auditory-only voice-identity recognition test, listeners were asked to recognise the identity of the six previously learned speakers in the four different levels of auditory noise. In each trial, participants viewed a centrally located white fixation cross while they listened to a speaker utter a two-word sentence.The name of one of the six learned speakers then immediately appeared onscreen. Participants were instructed to indicate via button press, "yes" or "no", whether the visually presented name matched the identity of the voice. The name was presented for maximum of 2 seconds and participants were free to respond at any time during the name presentation. A response triggered the offset of the current trial and the onset of the next trial. Alternatively, if a response was not made within 2 seconds, the next trial automatically began. Participants were encouraged to respond on each trial. Trials were interleaved with a 1 second inter-trial-interval (Figure 5).

\section{Auditory-only voice-identity recognition (sample trials)}

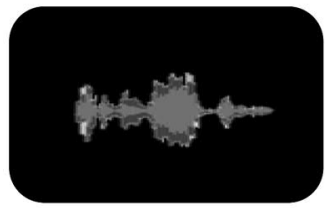

$\sim 1 \mathrm{sec}$

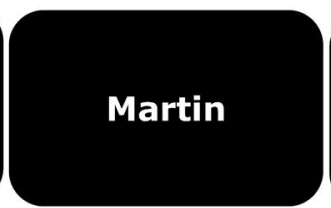

$\max 2 \mathrm{sec}$

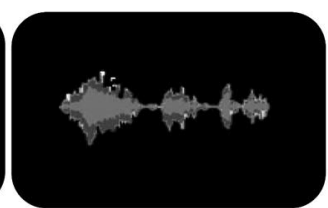

$\sim 1 \mathrm{sec}$

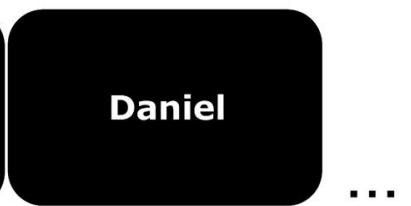

$\max 2 \mathrm{sec}$

time

Figure 5. Schematic illustration of the auditory-only voice-identity recognition test phase in Experiment 2. Following audio-visual training with six male speakers (procedure identical to Figure 2A), participants listened to blocks of sentences spoken by the same speakers in different levels of auditory noise $(-8,-4,0$, $+4 \mathrm{~dB}$ ). Each block contained 15 trials. In each trial, participants heard the speaker utter a two-word sentence which was followed immediately by the presentation of a name onscreen. The participant's task was to decide if the presented name matched the identity of the speaker or not. The trials were blocked by learning type (voice-face or voice-occupation) and noise level.

The auditory-only voice-identity recognition test phase consisted of 720 trials in total. For half the trials, the visually presented name was correct i.e., matched the identity of the voice. For the other half of the trials, it was incorrectly matched. Incorrect names were always taken from the same speaker set. The trials were blocked and presented in the same manner as described in Experiment 1. After 12 blocks participants were given a rest interval of 60 seconds. A brief practice period proceeded the auditory-only voice-identity recognition test. This practice period was identical to Experiment 1, with the exception that the task was to recognise the identity of the speakers. 


\section{Data Analysis}

As Experiment 1, trials in which the participant failed to make a response, i.e., missed trials, were disregarded from analysis ( $2.6 \%$ of all trials across participants, std. dev. $3.2 \%$; the min. \% of missed trials was $0 \%$, the max. was $12 \%$ ) and the overall trial count adjusted accordingly. Accuracy was calculated in an identical manner to Experiment 1: number of correct responses (voice-identity recognition) divided by the adjusted trial count, for each participant, for each condition (i.e., voice-face and voiceoccupation learning), per noise level. All other data including reaction times and the (normalised) face-benefit score, were calculated, and analysed, in an identical manner as Experiment 1. $\alpha$-level for significance and calculation of effect sizes are as in Experiment 1 . Mean accuracy and reaction time data can be viewed in Table 2.

\section{Results}

\section{Face-Benefit Score: Accuracy}

\section{Face-Benefit: Across Noise Levels}

In line with Experiment 1, not all participants displayed a face-benefit. Rather, there was an overall positive face-benefit across all noise levels for 19 of the 25 participants tested. A face-benefit of $76 \%$ of the participants is comparable to previous observations in neurotypical participants for voice-identity processing (von Kriegstein et al., 2008). The variability in the face-benefit across these 19 individuals was again large: e.g., in SNR -8 dB it ranged from $-2.2 \%$ to $28.8 \%$. Therefore, we again calculated the normalised face-benefit score for each participant, for each noise level (\% correct voice-face, minus, \% correct voice-occupation learned speakers for each noise level, divided by each individual's average face-benefit score i.e., the average across all noise levels). We investigated if the normalised face-benefit score increased in this group ( $\mathrm{N}$ =19) as a consequence of increasing auditory noise. In line with our hypothesis, there was a significant correlation with noise level i.e., the normalised face-benefit increased as the quality of the auditory signal decreased $(r=-0.24, p=0.038)$. The model results showed that an increase in noise level significantly predicted the normalised facebenefit score $(\Omega=-0.24, t(74)=-2.11, p=0.038)$ and in parallel explained a significant proportion of score variance, i.e., $6 \%\left(\mathrm{R}^{2}=0.06, \mathrm{~F}(1,74)=4.45, \mathrm{p}=0.038, \mathrm{n}^{2}=0.06\right)$. This systematic increase in the normalised face-benefit with increasing noise can be viewed in Figure 6A.

\section{Face-Benefit: Per Noise Level}

As Experiment 1, we conducted a categorical analysis of the face-benefit (nonnormalised scores) in each separate noise level. For the analyses with all participants ( $\mathrm{N}$ $=25$ ), the face-benefit was significant (face-benefit versus zero) in 3 of the 4 noise levels (see Table 2): SNR - $8 \mathrm{~dB}$ (M face-benefit $=7.18 \%)(\mathrm{t}(24)=3.11, \mathrm{p}=0.005,95 \%$ CI [2.41, 
11.94], $\mathrm{d}=0.62)$; SNR $-4 \mathrm{~dB}(\mathrm{M}$ face-benefit $=6.37 \%)(\mathrm{t}(24)=2.89, \mathrm{p}=0.007,95 \% \mathrm{CI}$ $[1.82,10.91], d=0.58)$; SNR $4 \mathrm{~dB}$ (M face-benefit $=5.73 \%)(\mathrm{t}(24)=2.62, \mathrm{p}=0.015,95 \%$ CI $[1.20,10.25], d=0.52)$; but not SNR $0 \mathrm{~dB}($ M face-benefit $=4.16 \%)(\mathrm{t}(24)=1.73, \mathrm{p}=$ $0.096,95 \%$ CI $[-0.79,9.13], d=0.35)$. All $\alpha$ levels for significance are Holm-Bonferroni adjusted for four comparisons. For the analysis restricted to the participants who showed an overall benefit for voice-face learning $(\mathrm{N}=19)$, the face-benefit was evident in every noise level (Holm-Bonferroni corrected): SNR -8 dB (M face-benefit $=11.49 \%$ ) $(\mathrm{t}(18)=5.62, \mathrm{p}<0.001,95 \%$ CI $[7.19,15.79], \mathrm{d}=1.29)$; SNR $-4 \mathrm{~dB}$ (M face-benefit $=$ 9.96\%) (t(18) $=4.45, \mathrm{p}<0.001,95 \%$ CI [5.25, 14.67], $\mathrm{d}=1.02)$; SNR $0 \mathrm{~dB}$ (M face-benefit $=8.64 \%)(\mathrm{t}(18)=4.01, \mathrm{p}<0.001,95 \% \mathrm{CI}[4.11,13.17], \mathrm{d}=0.92)$; SNR $4 \mathrm{~dB}(\mathrm{M}$ facebenefit $=8.58 \%)(t(18)=3.54, p=0.002,95 \%$ CI $[3.49,13.68], d=0.81)$. The face-benefit (non-normalised) is plotted in Figure 6B (see also Table 2). Note, all effects remained significant for the analyses with the normalised face-benefit scores for $\mathrm{N}=19$ (for the four noise levels - all ps <0.01, Figure 6A).

\section{Auditory-Only Voice-Identity Recognition Accuracy}

A

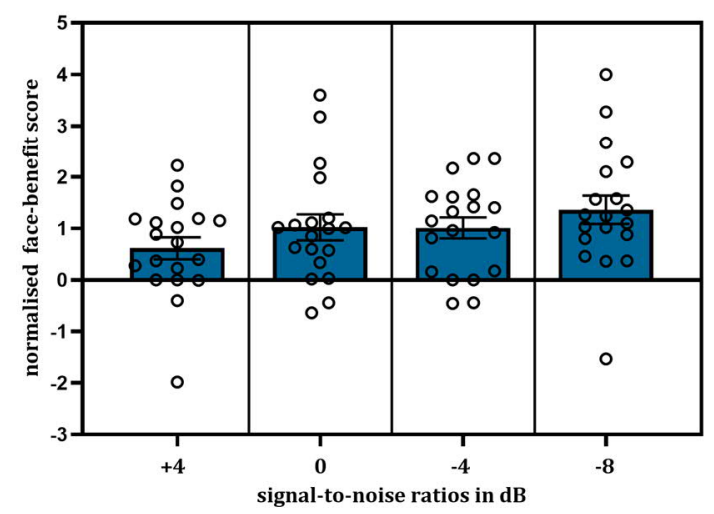

B

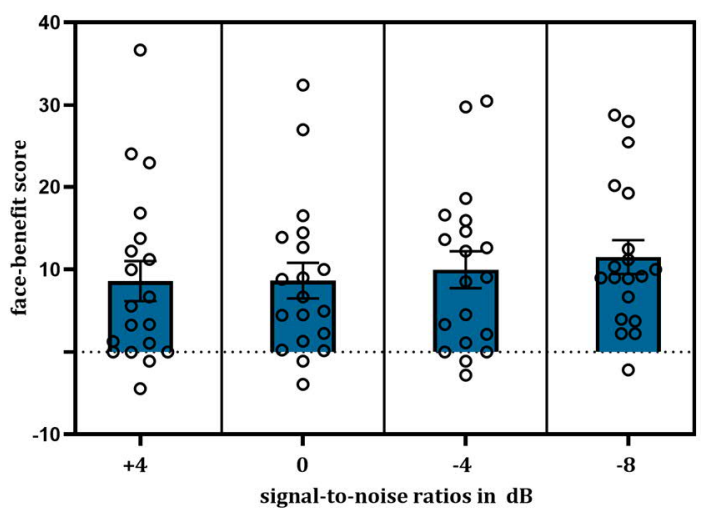

Figure 6. Plots showing the (A) normalised face-benefit and (B) face-benefit scores for voice-identity recognition across the increasing noise levels $(\mathrm{N}=19)$. In $(A)$ the increasing noise level significantly predicted the normalised face-benefit scores. In (A) and (B) the (normalised) face-benefit was evident in each of the noise levels (all ps < 0.01). In (A) and (B) error bars show \pm 1 standard error of the mean. Individual participant scores are displayed as single points.

Face-Benefit Score: Reaction Time

Face-Benefit Reaction Time: Across Noise Levels

For the linear regression analysis including all 25 participants, there was no evidence of a relationship between face-benefit RT scores and the auditory noise level $(\beta=0.13, t(98)=1.30, p=0.20)\left(R^{2}=0.017, F(1,98)=1.69, p=0.20, n_{p}^{2}=0.017\right)$. The analysis including only the participants $(\mathrm{N}=19)$ with a positive overall face-benefit (based on accuracy performance) revealed a similar result: In this group, there was also 
no relationship evident between face-benefit RTs and the noise level $(r=0.14, p=0.23)$. The noise level did not significantly predict $(\Omega=0.14, t(74)=1.22, \mathrm{p}=0.23)$ or explain a significant amount of variance in face-benefit RTs scores $\left(R^{2}=0.019, F(1,74)=1.50, p=\right.$ $\left.0.23, \mathrm{n}_{\mathrm{p}}^{2}=0.019\right)$, indicating no speed accuracy trade-off for the effect of learning, as a function of noise. Rather RTs appeared faster for voice-face, compared to voiceoccupation, learned speakers in the four noise levels (Figure 7) - this potential RT facebenefit was examined for each noise level in the next section.

Table 2. Auditory-only voice-identity recognition performance. Mean accuracy and reaction times scores (with standard deviations) for voice-face and voice-occupation learned speakers, in the four noise levels. Scores for all 25 participants are displayed on the left of the table, scores for the 19 participants with a positive overall face-benefit score (defined via accuracy) are to the right. All scores are derived from the non-normalised data.

\begin{tabular}{|c|c|c|c|c|c|c|c|c|}
\hline \multirow[b]{2}{*}{ signal-to-noise ratio } & \multicolumn{4}{|c|}{$\begin{array}{l}\text { All Participants } \\
(\mathrm{N}=25)\end{array}$} & \multicolumn{4}{|c|}{$\begin{array}{l}\text { Positive Face-Benefit Participants } \\
(\mathrm{N}=19)\end{array}$} \\
\hline & $+4 \mathrm{~dB}$ & $\mathbf{0 ~ d B}$ & $-4 \mathrm{~dB}$ & $-8 \mathrm{~dB}$ & $+4 \mathrm{~dB}$ & $\mathbf{0} \mathbf{d B}$ & $-4 \mathrm{~dB}$ & $-8 \mathrm{~dB}$ \\
\hline \multicolumn{9}{|l|}{ voice-face } \\
\hline \% Correct & $\begin{array}{l}89.72 \\
(8.40)\end{array}$ & $\begin{array}{l}87.10 \\
(10.61)\end{array}$ & $\begin{array}{l}86.52 \\
(8.45)\end{array}$ & $\begin{array}{l}84.06 \\
(10.44)\end{array}$ & $\begin{array}{l}91.74 \\
(5.34)\end{array}$ & $\begin{array}{l}90.08 \\
(7.57)\end{array}$ & $\begin{array}{l}88.27 \\
(6.44)\end{array}$ & $\begin{array}{l}86.88 \\
(6.61)\end{array}$ \\
\hline RT (milliseconds) & $\begin{array}{l}740.74 \\
(179.11)\end{array}$ & $\begin{array}{l}729.36 \\
(171.62)\end{array}$ & $\begin{array}{l}745.77 \\
(157.45)\end{array}$ & $\begin{array}{l}763.56 \\
(160.33)\end{array}$ & $\begin{array}{l}710.00 \\
(155.90)\end{array}$ & $\begin{array}{l}690.59 \\
(167.31)\end{array}$ & $\begin{array}{l}708.34 \\
(145.19)\end{array}$ & $\begin{array}{l}728.76 \\
(151.94)\end{array}$ \\
\hline \multicolumn{9}{|l|}{ voice-occupation } \\
\hline$\%$ Correct & $\begin{array}{l}83.99 \\
(12.03)\end{array}$ & $\begin{array}{l}82.93 \\
(10.56)\end{array}$ & $\begin{array}{l}80.15 \\
(11.37) \\
\end{array}$ & $\begin{array}{l}76.88 \\
(12.11)\end{array}$ & $\begin{array}{l}83.16 \\
(12.86)\end{array}$ & $\begin{array}{l}81.44 \\
(11.23)\end{array}$ & $\begin{array}{l}78.31 \\
(11.84)\end{array}$ & $\begin{array}{ll}75.38 \\
(12.70)\end{array}$ \\
\hline RT (milliseconds) & $\begin{array}{l}747.85 \\
(154.21) \\
\end{array}$ & $\begin{array}{l}753.07 \\
(145.83) \\
\end{array}$ & $\begin{array}{l}778.82 \\
(159.76) \\
\end{array}$ & $\begin{array}{l}810.52 \\
(152.05) \\
\end{array}$ & $\begin{array}{l}754.07 \\
(167.03) \\
\end{array}$ & $\begin{array}{l}752.99 \\
(145.82) \\
\end{array}$ & $\begin{array}{l}770.70 \\
(173.34) \\
\end{array}$ & $\begin{array}{l}809.60 \\
(158.38)\end{array}$ \\
\hline \multicolumn{9}{|l|}{ face-benefit } \\
\hline$\%$ Correct & $\begin{array}{l}5.73 \\
(10.96)\end{array}$ & $\begin{array}{l}4.16 \\
(12.02)\end{array}$ & $\begin{array}{l}6.37 \\
(11.00)\end{array}$ & $\begin{array}{l}7.18 \\
(11.54)\end{array}$ & $\begin{array}{l}8.58 \\
(10.57)\end{array}$ & $\begin{array}{l}8.64 \\
(9.40)\end{array}$ & $\begin{array}{l}9.96 \\
(9.77)\end{array}$ & $\begin{array}{l}11.49 \\
(8.92)\end{array}$ \\
\hline RT (milliseconds)* & $\begin{array}{l}-7.11 \\
(122.5)\end{array}$ & $\begin{array}{l}-23.71 \\
(114.16) \\
\end{array}$ & $\begin{array}{l}-33.05 \\
(113.93)\end{array}$ & $\begin{array}{l}-46.96 \\
(95.56)\end{array}$ & $\begin{array}{l}-44.06 \\
(91.83) \\
\end{array}$ & $\begin{array}{l}-62.39 \\
(97.01)\end{array}$ & $\begin{array}{l}-62.36 \\
(91.27)\end{array}$ & $\begin{array}{l}-80.84 \\
(74.21) \\
\end{array}$ \\
\hline
\end{tabular}

*negative reaction times (RT) indicate comparatively faster responses.

Face-Benefit Reaction Time: Per Noise Level

For the analyses including all $\mathrm{N}=25$ participants, when Holm-Bonferroni correcting for four comparisons, there was no significant face-benefit RT for voiceidentity recognition in any of the noise levels (all ps $>0.02$, see Table 2 for scores). For the 19 participants with a positive overall face-benefit based on accuracy performance, the analyses revealed a significant face-benefit RT (i.e., the face-benefit RT differed from zero) in every noise level tested (all $\alpha$ levels for significance Holm-Bonferroni adjusted). For SNR -8 dB the M face-benefit RT was -80.84 milliseconds $(\mathrm{t}(18)=-4.75, \mathrm{p}<0.001$, 95\% CI [-116.61, -45.07], $d=-1.09)$; SNR $-4 \mathrm{~dB}$ M face-benefit RT $=-62.36$ milliseconds $(\mathrm{t}(18)=-2.98, \mathrm{p}=0.008,95 \% \mathrm{CI}[-106.35,-18.38], \mathrm{d}=-0.68)$; SNR $0 \mathrm{~dB}$ M face-benefit $\mathrm{RT}=-62.39$ milliseconds ( $\mathrm{t}(18)=-2.80, \mathrm{p}=0.01,95 \%$ CI $[-109.15,-15.64], \mathrm{d}=-0.64$ ); $\mathrm{SNR}+4 \mathrm{~dB}$ M face-benefit RT $=-44.06$ milliseconds $(\mathrm{t}(18)=-2.09, \mathrm{p}=0.05,95 \%$ CI $[-$ 88.32, 0.19], $d=-0.48$ ); See Figure 7. 


\section{Auditory-Only Voice-Identity Recognition Reaction Time}

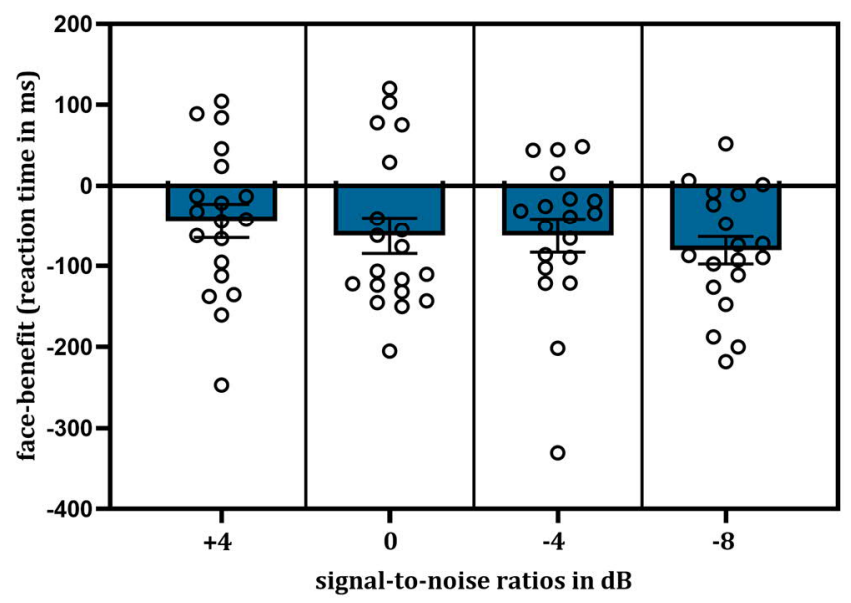

Figure 7. Plot of the face-benefit reaction time scores for voice-identity recognition $(N=19)$ for each of the SNR listening conditions. In each of the four noise levels responses were comparatively faster (indicated via negative RTs) for voice-face, compared to voice-occupation, learned speakers (all ps < 0.05). Error bars show \pm 1 standard error of the mean. Individual participant scores are displayed as single points.

\section{Discussion}

The face-benefit for voice-identity recognition was evident for the majority of participants tested i.e., 76\%.In these participants, the normalised face-benefit increased in parallel with the degree of noise in the auditory signal, suggesting a systematic benefit on sensory voice-identity processing for speakers learned by face. In line with Experiment 1, in the categorical analysis the (normalised) face-benefit was again evident in the noisiest listening condition i.e., SNR -8 dB. Moreover, it was evident in all other noise levels tested and was also reflected at the level of reaction time - voice-face learned speakers were consistently recognised faster in all noise levels $(\mathrm{N}=19)$. This was qualitatively different from the (normalised) face-benefit for speech recognition accuracy (Experiment 1 ), which was only significantly present at the lowest SNR $(-8 \mathrm{~dB})$. In the following main Discussion, we explore in more detail potentials for these similarities and differences between Experiment 1 and 2.

\section{General Discussion}

The current study had two key findings: we showed that for those who displayed a face-benefit, the (normalised) face-benefit for both auditory-only speech and voiceidentity recognition increased as the saliency of the auditory signal decreased. These findings are in line with our predictions and fit well with an audio-visual model of human auditory communication (von Kriegstein et al., 2008). In this view, learned visual mechanisms are behaviourally relevant for supporting the sensory processing of the auditory signal, providing useful constraints which help to predict and resolve what is 
heard in the auditory domain (Figure 1C). Our findings support this view and demonstrate for the first time that, even in the absence of concurrent visual input, the sensory system has developed a flexible mechanism to deal with auditory uncertainty learned visual mechanisms are recruited to enhance the recognition of the auditory signal, boosting recognition when the sensory information is weaker. This observation also speaks against strict auditory-only models of auditory processing (Figure 1B), which propose that recruitment of visual mechanisms is a by-product of successful auditory recognition. Under this view, one would not expect a face-benefit on auditory processing. Moreover, for it to increase as auditory processing becomes more challenging.

The Face-Benefit for Speech Increases in Noise: Parallels with Speech Processing with Concurrent Audio-Visual Input

In terms of speech recognition, the face-benefit was most evident in the noisiest listening condition - SNR -8 dB. In this noise level, the face-benefit was approximately four-fold $(\sim 4.6 \%)$ what had been observed in any other SNR tested (all $<1 \%)$. This large increase at a specific SNR parallels previous studies on the effect of concurrent visual input on speech recognition in noise (Ross et al., 2007; Liu et al., 2013). These studies, which have parametrically altered the noise level in the auditory signal, have shown that while the benefit of visual input either increases linearly (Ross et al., 2007; Ma et al., 2009) or remains relatively consistent ( $\mathrm{Liu}, 2007)$ with increasing noise at lower noise levels (up to SNR $-8 \mathrm{~dB}$ ), there is a "special zone" (Ross et al., 2007) where the multisensory benefit on speech recognition is maximum (Ross et al., 2007; Ma et al., 2009; Liu et al., 2013). This maximum or sudden increase has been observed for what has been labelled as "intermediate" SNRs. For example, when the range of auditory SNRs tested spans from $0 \mathrm{~dB}$ to $-16 \mathrm{~dB}$ or $-24 \mathrm{~dB}$, the benefit has been maximum at $-12 \mathrm{~dB}$, after which it tails off substantially with increasing noise levels.

The special zone for audio-visual speech recognition is contrary to what would be predicted based on a general principle of multisensory integration, i.e., inverse effectiveness (Meredith and Stein, 1986). Inverse effectiveness proposes that the benefit of concurrent multiple sensory inputs on perception should be greatest when the quality of the unisensory input is poorest i.e., at the lowest auditory SNR. Earlier reported audiovisual speech recognition studies supported this position (Sumby and Pollack, 1954; Erber, 1969). For example, Sumby and Pollack (1954) reported this inverse relationship at auditory SNRs up to -30 dB. However, Ross et al.(2007) have argued that the observed benefits at these lower SNRs were likely driven by artefacts of the task design. In Sumby and Pollack's study, participants were exposed to checklists, prior to and during testing, which contained the spoken words. This may have allowed the participant to guess the words at lower SNRs based on speech reading performance, leading to inflated audiovisual effects at these SNRs. Rather, Ross et al. (2007) argue that the sensory system is tuned to integrate multiple sensory inputs for speech at more intermediate SNRs (Ross et al., 2007). In contrast, at the absolute extremes the system would rely on unisensory 
processing - visual-only (lip-reading) processing when the auditory signal is severely degraded (lowest SNRs) or auditory-only when the auditory signal is highly salient (highest SNRs). In parallel to the special zone for audio-visual input, it is possible that the face-benefit for speech recognition in auditory-only conditions may also be maximal for these selective SNRs, i.e., in our study at - $8 \mathrm{~dB}$. At these specific zones engagement of the visual system, and the behavioural face-benefit, may be maximally stimulated. Potentially, at lower noise levels the system may be less reliant on additional visual mechanisms and may explain why the previously observed face-benefits for speech in more optimal listening conditions have been relatively small (gains between $0.43 \%$ (n.s.) - 2.08 \% (von Kriegstein et al., 2008; Schelinski, Riedel and von Kriegstein, 2014; Riedel et al., 2015).

Differences in the Visual Mechanisms Underpinning the Face-Benefit for Speech and Voice-identity Recognition

Paralleling speech recognition, the face-benefit for voice-identity recognition was also largest in the noisiest listening condition - SNR -8 dB. Previous studies have demonstrated that manipulations of concurrent visual facial input can affect voiceidentity recognition (Schweinberger, Robertson and Kaufmann, 2007; Robertson and Schweinberger, 2010; Schweinberger and Robertson, 2017). However, to the best of our knowledge, no study has yet explored how concurrent visual input affects voice-identity recognition in noisy listening conditions, making it difficult to directly compare whether the benefit of visual information on speech and voice-identity processing is equatable.

Notably, our results showed that the recognition of speakers learned by face was consistently superior, i.e., present for both accuracy and reaction times and evident in all noise levels including those with a relatively high SNR, to those learned in the control condition. Potentially, there may be differences in voice-identity and speech processing which may reflect such findings - Voice-identity recognition is inferior to our ability to recognise others by face (Ellis, Jones and Mosdell, 1997; Hanley, Smith and Hadfield, 1998; Hanley and Turner, 2000; Hanley and Damjanovic, 2009; review see Stevenage and Neil, 2014). It is therefore likely that interactions between the voice and face systems fundamentally support familiar voice-identity recognition (Jones and Tranel, 2001; von Kriegstein, Kleinschmidt and Giraud, 2006; Maguinness and von Kriegstein, 2017). These cross-modal effects may therefore be more readily observed for voiceidentity recognition, even at lower SNRs, when the auditory signal is relatively salient (Sheffert and Olson, 2004; von Kriegstein et al., 2008; Schall et al., 2013; Schelinski, Riedel and von Kriegstein, 2014). Indeed, the face-benefit for voice-identity is a robust phenomenon which has been observed under more optimal listening conditions in several studies which have employed numerous audio-visual control learning conditions. These control conditions have included familiarising the listener with the speaker by voice alone (Sheffert and Olson, 2004; Zäske, Mühl and Schweinberger, 2015) or in conjunction with other visual input including the name (von Kriegstein and Giraud, 2006) or, as in the current study, a visual image depicting the speaker's 
occupation (von Kriegstein et al., 2008; Schall et al., 2013; Schelinski, Riedel and von Kriegstein, 2014). This robust benefit of voice-face learning is likely underpinned by perceptual system's sensitivity to the common-cause static identity cues available in both sensory streams. Voices are caused by physical visual structures (i.e., the vocal tract) and static voice properties including vocal-tract resonance (i.e., timbre) and fundamental frequency (i.e., pitch) provide information about the visual structural characteristics of the speaker, including face-identity (Krauss, Freyberg and Morsella, 2002; Ives, Smith and Patterson, 2005; Smith and Patterson, 2005; Smith et al., 2005; Ghazanfar et al., 2007; Mavica and Barenholtz, 2013; Smith et al., 2016; Smith et al., 2016; Kim et al., 2019; Oh et al., 2019). These causal cross-modal relationships are rapidly acquired (Shams and Seitz, 2008; von Kriegstein et al., 2008), facilitating subsequent auditory-only recognition processing at a speaker specific level (Blank, Kiebel and von Kriegstein, 2015).

In contrast, the face-benefit for speech recognition has been less investigated and when observed tends to be smaller than that observed for voice-identity recognition (von Kriegstein et al., 2008; Schelinski, Riedel and von Kriegstein, 2014; Riedel et al., 2015), as in the present study where benefits of $<1 \%$ were observed in all noise levels, except for SNR -8 dB. While it is possible that the engagement of the visual system may be less apparent for speech recognition at lower SNRs, it is also important to consider that these small face-benefits $(<1 \%)$ may also be expected given the high recognition rates in these lower noise levels, where recognition performance was markedly stable and robust ( $>97 \%$ correct, Table 1). This robust performance may have been influenced by the SNR ranges tested - potentially they may not have been challenging enough to impact speech recognition. Employing a lower SNR (-10 dB), however, led to chance performance in pilot experiments when participants were exposed to one noise level during auditory-only testing. Another possibility is that recognition performance may have been inflated due to sentence repetition. All 30 sentences were heard in each of the different noise levels. Potentially, if a sentence has been heard in a high SNR, then when it is repeated in a lower SNR it may still be clearly audible. However, this audibility may be driven by top-down factors (i.e., a general priming effect), thus making it more difficult to detect task effects of interest i.e., learning condition. Additionally, the audiovisual training paradigm was focused on recognising the identity of the speaker. Potentially, training which emphasises processing of the dynamic speech signal may also impact subsequent speech recognition performance for face learned speakers.

\section{Inter-Individual Variability in the Face-Benefit}

While the face-benefit appears to be a supportive mechanism for auditory-only processing, it is important to consider that not all individuals displayed a face-benefit. And the increase in the face-benefit, for both speech and voice-identity recognition, with noise was restricted to those who benefitted overall (i.e., average benefit across noise levels) from voice-face learning. The number of participants who showed this overall face-benefit, in the separate speech and voice-identity recognition tasks, paralleled 
previous observations (von Kriegstein et al., 2008). Currently, it is unclear why some individuals do not benefit from this multisensory learning. In general, individual differences have been observed in a range of multisensory tasks, when concurrent sensory cues are available (de Haas et al., 2012; Gurler et al., 2015; Mallick, Magnotti and Beauchamp, 2015; Hirst et al., 2020). Prior observations in neurotypicals suggest no correlation between the face-benefits for speech and voice-identity recognition within participants (von Kriegstein et al., 2008) (e.g., a participant with a high face-benefit for speech will not necessarily also exhibit a high face-benefit for voice-identity recognition), indicating that variability in the face-benefit is unlikely to be governed by a general mechanism. Rather, findings from autism spectrum disorder suggest that the face-benefit for auditory-only speech processing may relate to differences in lip-reading abilities (von Kriegstein, Kleinschmidt and Giraud, 2006; von Kriegstein et al., 2008; Maguinness and von Kriegstein, 2017). While findings from developmental prosopagnosia (McConachie, 1976), i.e., a severe deficit in face-identity processing, implicate intact face-identity processing as a necessary component for the face-benefit on voice-identity processing (von Kriegstein, Kleinschmidt and Giraud, 2006; von Kriegstein et al., 2008; Maguinness and von Kriegstein, 2017).

Such findings imply that variability in specific visual abilities may potentially relate to the inter-individual differences observed in the current study. For example, the ability to perceive the lip-movements of a specific speaker likely translates into how precise the generative model for this specific speaker will be. We speculate that participants with a less precise generative model about the speaker-specific articulatory dynamics, will profit less from this predictive information, in the context of auditoryonly processing. In the current study we did not collect direct measures of lip-reading or face-identity recognition performance, additionally, participants were novel in each of the two tasks. Further studies examining these measures across sensory modalities may assist in determining the means by which this inter-individual variability may arise in the face-benefit and if, or how, it may vary as a function of auditory noise.

\section{The Face-Benefit: Facilitating Recognition Beyond Auditory Noise}

Challenging listening conditions surround us daily, such as when we speak with someone on the phone in the presence of background noise, or in the context of multiple speakers. Listening conditions are not only challenging due to extrinsic factors; auditory processing can also be impacted due to developmental disorders (e.g., developmental dyslexia or auditory processing disorder) or advancing age (Pichora-Fuller and Souza, 2003; Ziegler et al., 2009; Roth, Hanebuth and Probst, 2011; Iliadou et al., 2017). Indeed, one of the most commonly reported perceptual problems in older adults is difficultly in processing speech, particularly when listening environments are challenging (Sommers, 1997; Schneider et al., 2002; Surprenant, 2007; Pichora-Fuller, 2008; Sheldon et al., 2008). Our results suggest that in a similar vein to concurrent visual input (Sommers, Tye-Murray and Spehar, 2005; Maguinness et al., 2011), auditory processing in such groups may also benefit from audio-visual voice-face learning and may help resolve 
noisy auditory input. Several studies have demonstrated that older (Holmes, Domingo and Johnsrude, 2018), and also younger (Kreitewolf, Mathias and von Kriegstein, 2017), adults are more accurate at recognising speech utterances from familiar talkers (Johnsrude et al., 2013) and this benefit is greatest in adverse listening conditions (Souza et al., 2013). It is possible that this gain may be mediated by the recruitment of additional visual mechanisms (in cases where the familiar speakers were also known by face) which may assist in enhancing the predictability of the auditory signal.

How is the Face-Benefit in Noise Represented in the Brain?

Currently, the precise neural mechanisms for the face-benefits for speech and voice-identity recognition in noise are unknown. However, it is conceivable that they may be underpinned by enhanced recruitment of the visual regions which mediate these benefits in more optimal listening conditions i.e., FFA for voice-identity recognition and the left pSTS-mFA for speech recognition (von Kriegstein et al., 2008; Schall et al., 2013; Schall and von Kriegstein, 2014). Previous findings have shown that FFA responses during voice-identity recognition for speakers known by face occur $\sim 110 \mathrm{~ms}$ after auditory onset (Schall et al., 2013), a time point when voice-identity recognition has yet to occur in the human brain (Schweinberger, 2001). Such early responses suggest that visual mechanisms in the FFA play a role in the sensory processing of the voice. Indeed, in that MEG study, prior voice-face learning modulated the auditory processing of the voice, with a temporal facilitation of the M200 auditory evoked field component, which correlated with listeners' face-benefit scores. Conceivably, enhanced responses in these visual regions would provide increased constraints for processing the auditory signal in noise i.e., visual regions feeding back information to the auditory system. Enhanced connectivity between the respective visual and auditory regions during auditory-only processing in noisy listening conditions may be observed.

\section{Conclusion}

In summary, we replicated the face-benefit for speech and voice-identity recognition observed in previous studies and showed, for the first time, that visual information acquired during audio-visual learning is used in an adaptable manner, tailored to task demands, to enhance subsequent auditory-only recognition in challenging listening conditions. These findings advance and corroborate an audiovisual model of human auditory communication and suggest that the brain has developed a remarkably flexible mechanism for enhancing auditory processing considering sensory demands. 


\section{References}

Arnal, L. H. et al. (2009) 'Dual neural routing of visual facilitation in speech processing.', The Journal of Neuroscience, 29, pp. 13445-13453. doi: 10.1523/ JNEUROSCI.3194-09.2009.

Axelrod, V. and Yovel, G. (2015) 'Successful decoding of famous faces in the fusiform face area', PLoS ONE, 10(2), p. e0117126. doi: 10.1371/journal.pone.0117126.

Blank, H., Kiebel, S. J. and von Kriegstein, K. (2015) 'How the human brain exchanges information across sensory modalities to recognize other people', Human Brain Mapping, (36), pp. 324-339.

Bourguignon, M. et al. (2020) 'Lip-reading enables the brain to synthesize auditory features of unknown silent speech', Journal of Neuroscience, 40(5), pp. 10531065. doi: 10.1523/ JNEUROSCI.1101-19.2019.

Bunzeck, N. et al. (2005) 'Scanning silence: Mental imagery of complex sounds', NeuroImage, 26(4), pp. 1119-1127. doi: 10.1016/j.neuroimage.2005.03.013.

Campbell, R. (2008) 'The processing of audio-visual speech: empirical and neural bases.', Philosophical transactions of the Royal Society of London. Series B, Biological sciences, 363(1493), pp. 1001-1010. doi: 10.1098/ rstb.2007.2155.

Chandrasekaran, C. et al. (2009) The natural statistics of audiovisual speech', PLoS Computational Biology, 5(7). doi: 10.1371/journal.pcbi.1000436.

Chandrasekaran, C. and Ghazanfar, A. A. (2013) 'Coding across sensory modalities: Integrating the dynamic face with the voice', in Principles of Neural Coding. CRC Press.

Cohen, J. (1969) Statistical Power Analysis for the Behavioural Sciences. New York: Academic Press.

Cohen, J. (1988) Statistical Power Analysis for the Behavioral Sciences. New York: Routledge.

Crosse, M. J., Di Liberto, G. M. and Lalor, E. C. (2016) 'Eye can hear clearly now: Inverse effectiveness in natural audiovisual speech processing relies on long-term crossmodal temporal integration', Journal of Neuroscience, 36(38), pp. 98889895. doi: 10.1523/ JNEUROSCI.1396-16.2016.

Davis, C. et al. (2008) The effect of viewing speech on auditory speech processing is different in the left and right hemispheres.', Brain Research. Elsevier B.V., 1242, pp. 151-161. doi: 10.1016/j.brainres.2008.04.077.

Eger, E., Schyns, P. G. and Kleinschmidt, A. (2004) 'Scale invariant adaptation in fusiform face-responsive regions', NeuroImage, 22, pp. 232-242. doi: 10.1016/j.neuroimage.2003.12.028.

Ellis, H. D., Jones, D. M. and Mosdell, N. (1997) 'Intra- and inter-modal repetition priming of familiar faces and voices', British Journal of Developmental Psychology, 88, pp. 143-156.

Erber, N. P. (1969) 'Interaction of Audition and Vision in the Recognition of Oral Speech Stimuli', Journal of Speech, Language, and Hearing Research, 12(2), pp. 423-425.

Ewbank, M. P. and Andrews, T.J. (2008) 'Differential sensitivity for viewpoint between 
familiar and unfamiliar faces in human visual cortex', NeuroImage, 40(4), pp. 1857-1870.

Ghazanfar, A. A. et al. (2007) Vocal-Tract Resonances as Indexical Cues in Rhesus Monkeys', Current Biology. Elsevier Ltd, 17(5), pp. 425-430. doi: 10.1016/j.cub.2007.01.029.

Grant, K. W. and Seitz, P. (2000) The use of visible speech cues for improving auditory detection', Journal of the Acoustical Society of America, 108(3), pp. 1197-1208.

Grill-Spector, K., Knouf, N. and Kanwisher, N. (2004) The fusiform face area subserves face perception, not generic within-category identification.', Nature Neuroscience, 7(5), pp. 555-562. doi: 10.1038/ nn1224.

Gurler, D. et al. (2015) 'A link between individual differences in multisensory speech perception and eye movements', Attention, Perception, \& Psychophysics. doi: 10.3758/ s13414-014-0821-1.

de Haas, B. et al. (2012) 'Grey matter volume in early human visual cortex predicts proneness to the sound-induced flash illusion', Proceedings of the Royal Society B: Biological Sciences, 279(1749), pp.4955-4961. doi: 10.1098/rspb.2012.2132.

Hanley,J. R. and Damjanovic, L. (2009) 'It is more difficult to retrieve a familiar person's name and occupation from their voice than from their blurred face.', Memory (Hove, England), 17(8), pp. 830-9. doi: 10.1080/ 09658210903264175.

Hanley, J. R., Smith, S. T. and Hadfield, J. (1998) 'I recognise you but I can't place you. An investigation of familiar-only experiences during tests of voice and face recognition', The Quaterly Journal of Experimental Psychology, 51A(1), pp. 179195.

Hanley, J. R. and Turner, J. M. (2000) Why are familiar-only experiences more frequent for voices than for faces?', The Quarterly Journal of Experimental Psychology Section A, 53A(4), pp. 1105-1116.

Hickok, G. and Poeppel, D. (2007) 'The cortical organization of speech processing', Nat.Rev.Neurosci., 8(5), pp. 393-402.

Hirst, R. J. et al. (2020) What you see is what you hear: Twenty years of research using the Sound-Induced Flash Illusion', Neuroscience and Biobehavioral Reviews. Elsevier Ltd, 118, pp. 759-774. doi: 10.1016/ j.neubiorev.2020.09.006.

Holm, S. (1979) 'A Simple Sequentially Rejective Multiple Test Procedure', Scandinavian Journal of Statistics, 6(2), pp. 65-70.

Holmes, E., Domingo, Y. and Johnsrude, I. S. (2018) 'Familiar voices are more intelligible, even if they are not recognized as familiar', Psychological Science, 29(10), pp. 1575-1583.

Iliadou, V. et al. (2017) 'A European perspective on auditory processing disordercurrent knowledge and future research focus', Frontiers in Neurology. Frontiers Media S.A., 8, p. 622. doi: 10.3389/ fneur.2017.00622.

Ives, D. T., Smith, D. R. and Patterson, R. D. (2005) 'Discrimination of speaker size from syllable phrases', The Journal of the Acoustical Society of America. 2006/ 01/20, 118(6), pp. 3816-3822. Available at: http:/ / www.ncbi.nlm.nih.gov/ pubmed/ 16419826.

Jääskeläinen, I. P. et al. (2004) 'Adaptation of neuromagnetic N1 responses to phonetic 
stimuli by visual speech in humans.', NeuroReport, 15(18), pp. 2741-2744.

Johnsrude, I. S. et al. (2013) 'Swinging at a cocktail party: voice familiarity aids speech perception in the presence of a competing voice.', Psychological Science, 24(10), pp. 1995-2004. doi: 10.1177/ 0956797613482467.

Jones, R. D. and Tranel, D. (2001) 'Severe developmental prosopagnosia in a child with superior intellect', Journal of Clinical and Experimental Neuropsychology, 23(3), pp. 265-273. doi: 10.1076/jcen.23.3.265.1183.

Kanwisher, N., McDermott, J. and Chun, M. M. (1997) The fusiform face area: A module in human extrastriate cortex specalized for face perception', The Journal of Neuroscience, 17(11), pp.4302-4311.

Kanwisher, N. and Yovel, G. (2006) 'The fusiform face area: a cortical region specialized for the perception of faces', Philosophical Transactions of the Royal Society B, 361(1476), pp. 2109-2128. doi: 10.1098/ rstb.2006.1934.

Kiebel, S.J., Daunizeau, J. and Friston, K.J. (2009) 'Perception and hierarchical dynamics', Frontiers in Neuroinformatics, 3, p. 20. Available at: http:/ / www.ncbi.nlm.nih.gov/ entrez/ query.fcgi?cmd=Retrieve\&db=PubMed\& dopt=Citation\&list_uids $=19649171$.

Kim, C. et al. (2019) 'On learning associations of faces and voices', in C., J. et al. (eds) Computer Vision - ACCV 2018. ACCV 2018. Lecture Notes in Computer Science. vol 11365. Springer, Cham, pp. 276-292. doi: 10.1007/978-3-030-20873-8_18.

Krauss, R. M., Freyberg, R. and Morsella, E. (2002) 'Inferring speakers' physical attributes from their voices', Journal of Experimental Social Psychology, 38, pp. 618-625.

Kreitewolf, J., Mathias, S. R. and von Kriegstein, K. (2017) 'Implicit Talker Training Improves Comprehension of Auditory Speech in Noise', Frontiers in Psychology, 8(September), pp. 1-8. doi: 10.3389/ fpsyg.2017.01584.

von Kriegstein, K. et al. (2005) 'Interaction of face and voice areas during speaker recognition', Journal of Cognitive Neuroscience, 17(3), pp. 367-376. doi: $10.1162 / 0898929053279577$.

von Kriegstein, K. et al. (2008) 'Simulation of talking faces in the human brain improves auditory speech recognition.', Proceedings of the National Academy of Sciences, 105(18), pp. 6747-6752. doi: 10.1073/ pnas.0710826105.

von Kriegstein, K. (2012) 'A multisensory perspective on human communication', in Murray, M. M. and Wallace, M.T. (eds) The Neural Bases of Multisensory Processes. CRC Press/ Taylor \& Francis.

von Kriegstein, K. and Giraud, A.-L. (2006) 'Implicit multisensory associations influence voice recognition', PLoS Biology, 4(10), p. e326. doi: 10.1371/ journal.pbio.0040326.

von Kriegstein, K., Kleinschmidt, A. and Giraud, A.-L. (2006) 'Voice recognition and cross-modal responses to familiar speakers' voices in prosopagnosia.', Cerebral Cortex, 16(9), pp. 1314-1322. doi: 10.1093/ cercor/ bhj073.

Liu, B. et al. (2013) 'Correlation between audio-visual enhancement of speech in different noise environments and SNR: A combined behavioral and electrophysiological study', Neuroscience. IBRO, 247, pp. 145-151. doi: 
10.1016/j.neuroscience.2013.05.007.

Liu, J., Harris, A. and Kanwisher, N. (2010) 'Perception of face parts and face configurations: An FMRI study', Journal of Cognitive Neuroscience, 22(1), pp. 203-211.

$\mathrm{Liu}, \mathrm{T}$. (2007) 'Learning sequence of views of three-dimensional objects: the effect of temporal coherence on object memory', Perception, 36(9), pp. 1320-1333. doi: $10.1068 / \mathrm{p} 5778$.

Ma, W. J. et al. (2009) 'Lip-Reading Aids Word Recognition Most in Moderate Noise: A Bayesian Explanation Using High-Dimensional Feature Space', PLoS ONE, 4(3), p. 4638. doi: 10.1371/journal.pone.0004638.

Maguinness, C. et al. (2011) 'The effect of combined sensory and semantic components on audio-visual speech perception in older adults.', Frontiers in Aging Neuroscience, 3, p. 19.

Maguinness, C. and von Kriegstein, K. (2017) 'Cross-modal processing of voices and faces in developmental prosopagnosia and developmental phonagnosia', Visual Cognition. Taylor \& Francis, 25(4-6), pp. 644-657. doi: 10.1080/13506285.2017.1313347.

Mallick, D. B., Magnotti, J.F. and Beauchamp, M. S. (2015) Variability and stability in the McGurk effect: contributions of participants, stimuli, time, and response type', Psychonomic Bulletin \& Review, 22(5), pp. 1299-1307. doi: 10.3758/s13423015-0817-4.Variability.

Mavica, L. W. and Barenholtz, E. (2013) 'Matching voice and face identity from static images.', Journal of Experimental Psychology: Human Perception and Performance, 39(2), pp. 307-312. doi: 10.1037/a0030945.

McConachie, H. R. (1976) 'Developmental prosopagnosia. A single case report', Cortex, 12(1), pp. 76-82. doi: 10.1016/ S0010-9452(76)80033-0.

Meredith, M.A. and Stein, B.E. (1986) 'Visual, auditory, and somatosensory convergence on cells in superior colliculus results in multisensory integration', Journal of Neurophysiology, 56, pp. 640-662.

Oh, T. H. et al. (2019) 'Speech2Face: Learning the face behind a voice', in Proceedings of the IEEE Computer Society Conference on Computer Vision and Pattern Recognition. IEEE Computer Society, pp. 7531-7540. doi: 10.1109/ CVPR.2019.00772.

Oldfield, R. C. (1971) The assessment and analysis of handedness: The edinburgh inventory', Neuropsycholgia, 9, pp. 97-113.

Peelle, J. E. and Sommers, M. S. (2015) 'Prediction and constraint in audiovisual speech perception', Cortex. Elsevier Ltd, 68, pp. 169-181. doi: 10.1016/j.cortex.2015.03.006.

Pichora-Fuller, M. K. and Souza, P. E. (2003) 'Effects of aging on auditory processing of speech.', International journal of audiology, 42 Suppl 2, pp. 2S11-6. Available at: http:/ / www.ncbi.nlm.nih.gov/ pubmed/ 12918623.

Richardson,J.T.E. (2011) 'Eta squared and partial eta squared as measures of effect size in educational research', Educational Research Review. Elsevier Ltd, 6(2), pp. 135-147. doi: 10.1016/j.edurev.2010.12.001. 
Riedel, P. et al. (2015) Visual face-movement sensitive cortex is relevant for auditoryonly speech recognition', Cortex, 68, pp. 86-99. doi: 10.1016/j.cortex.2014.11.016.

Robertson, D. M. C. and Schweinberger, S. R. (2010) The role of audiovisual asynchrony in person recognition.', Quarterly Journal of Experimental Psychology, 63(1), pp. 23-30.

Rosenblum, L. D., Johnson, J. A. and Saldana, H. M. (1996) 'Visual kinematic information for embellishing speech in noise', Journal of Speech and Hearing Research, 39(6), pp. 1159-1170.

Ross, L. a et al. (2007) 'Do you see what I am saying? Exploring visual enhancement of speech comprehension in noisy environments.', Cerebral Cortex, 17(5), pp. 1147-1153. doi: 10.1093/ cercor/ bhl024.

Roth, T. N., Hanebuth, D. and Probst, R. (2011) 'Prevalence of age-related hearing loss in Europe: a review.', European Archives of Otorhinolaryngology, 268(8), pp. 11011107. doi: 10.1007/s00405-011-1597-8.

Rotshtein, P. et al. (2005) 'Morphing Marilyn into Maggie dissociates physical and identity face representations in the brain.', Nature neuroscience, 8(1), pp. 107113. doi: $10.1038 / \mathrm{nn} 1370$.

Schall, S. et al. (2013) 'Early auditory sensory processing of voices is facilitated by visual mechanisms', NeuroImage. Elsevier Inc., 77, pp. 237-245. doi: 10.1016/j.neuroimage.2013.03.043.

Schall, S. et al. (2014) Voice identity recognition: Functional division of the right STS and its behavioural relevance', Journal of Cognitive Neuroscience, 27(2), pp. 280291.

Schall, S. and von Kriegstein, K. (2014) 'Functional connectivity between face-movement and speech-intelligibility areas during auditory-only speech perception.', PLoS ONE, 9(1), p. e86325. doi: 10.1371/journal.pone.0086325.

Schelinski, S., Riedel, P. and von Kriegstein, K. (2014) 'Visual abiltites are important for auditory-only speech recognition: Evidence from autism spectrum disorder', Neuropsychologia, 65, pp. 1-11.

Scholes, C., Skipper, J. and Johnston, A. (2020) 'The inter-relationship between the face and vocal-tract configuration during audio-visual speech', Proceedings of the National Academy of Sciences. doi: 10.1073/ pnas.2006192117.

Schweinberger, S. R. (2001) 'Human brain potential correlates of voice priming and voice recognition.', Neuropsychologia, 39(9), pp. 921-936. Available at: http:// www.ncbi.nlm.nih.gov/ pubmed/ 11516445 .

Schweinberger, S. R., Robertson, D. and Kaufmann,J. M. (2007) 'Hearing facial identities', The Quarterly Journal of Experimental Psychology, 60(10), pp. 1446-1456. doi: 10.1080/17470210601063589.

Schweinberger, S. R. and Robertson, D. M. C. (2017) 'Audiovisual integration in familiar person recognition', Visual Cognition. Taylor \& Francis, 0(0), pp. 1-22. doi: 10.1080/13506285.2016.1276110.

Shams, L. and Seitz, A. R. (2008) 'Benefits of multisensory learning.', Trends in Cognitive Sciences, 12(11), pp.411-417. doi: 10.1016/j.tics.2008.07.006. 
Sheffert, S. M. and Olson, E. (2004) 'Audiovisual speech facilitates voice learning.', Perception \& Psychophysics, 66(2), pp. 352-362. Available at: http:// www.ncbi.nlm.nih.gov/ pubmed/ 15129754.

Smith, D. R. R. et al. (2005) 'The processing and perception of size information in speech sounds', J.Acoust.Soc.Am., 117(1), pp. 305-318.

Smith, D. R. R. and Patterson, R. D. (2005) 'The interaction of glottal-pulse rate and vocaltract length in judgements of speaker size, sex and age', Journal of the Acoustical Society of America, 118, pp. 3177-3186.

Smith, H. M. J. et al. (2016) 'Concordant Cues in Faces and Voices: Testing the Backup Signal Hypothesis', Evolutionary Psychology, 14(1), pp. 1-10. doi: $10.1177 / 1474704916630317$.

Smith, H M J et al. (2016) 'Matching novel face and voice identity using static and dynamic facial images', Attention, Perception, \& Psychophysics, 78(3), pp. 868879. doi: 10.3758/ s13414-015-1045-8.

Sommers, M. S., Tye-Murray, N. and Spehar, B. (2005) 'Auditory-visual speech perception and auditory-visual enhancement in normal-hearing younger and older adults.', Ear and hearing, 26(3), pp. 263-275. Available at: http:// www.ncbi.nlm.nih.gov/ pubmed/ 15937408 .

Souza, P. et al. (2013) 'The advantage of knowing the talker', Journal of the American Academy of Audiology, 24(8), pp. 689-700. doi: 10.3766/ jaaa.24.8.6.

Stevenage, S. V and Neil, G. J. (2014) 'Hearing faces and seeing voices: The integration and interaction of face and voice processing', Psychologica Belgica, 54(3), pp. 266-281.

Sumby, W. H. and Pollack, I. (1954) 'Visual contribution of speech intelligibility in noise', The Journal of the Acoustical Society of America, 26(2), pp. 212-215.

Van Wassenhove, V., Grant, K. W. and Poeppel, D. (2005) 'Visual speech speeds up the neural processing of auditory speech', Proceedings of the National Academy of Sciences of the United States of America, 102(4), pp. 1181-1186. doi: $10.1073 /$ pnas. 0408949102 .

Weibert, K. and Andrews, T. J. (2015) 'Activity in the right fusiform face area predicts the behavioural advantage for the perception of familiar faces', Neuropsychologia. Elsevier, 75, pp. 588-596. doi: 10.1016/j.neuropsychologia.2015.07.015.

Young, A. W., Frühholz, S. and Schweinberger, S. R. (2020) 'Face and voice perception: Understanding commonalities and differences', Trends in Cognitive Sciences. Elsevier Ltd, 24(5), pp. 398-410. doi: 10.1016/j.tics.2020.02.001.

Zäske, R., Mühl, C. and Schweinberger, S. R. (2015) 'Benefits for voice learning caused by concurrent faces develop over time', PLoS ONE, 10(11), pp. 1-12. doi: 10.1371/journal.pone.0143151.

Ziegler,J.C. et al. (2009) 'Speech-perception-in-noise deficits in dyslexia', Developmental Science. John Wiley \& Sons, Ltd, 12(5), pp. 732-745. doi: 10.1111/j.14677687.2009.00817.x. 


\section{Acknowledgements}

We thank Laura Smith for her work on the project including data collection and preparation. We also thank Nicola Frasson for help with additional data collection. This work was funded by a Max Planck Research Group grant to KvK. CM also receives funding from the ERC-consolidator grant SENSOCOM 647051 and the DFG KR 3735/ 51.

The authors declare no competing financial interests. 